\title{
2D Navier-Stokes equation with cylindrical fractional Brownian noise
}

\author{
Benedetta Ferrario $^{1}$. Christian Olivera ${ }^{2}$
}

Received: 26 February 2018 / Accepted: 12 November 2018 / Published online: 11 December 2018 (c) Fondazione Annali di Matematica Pura ed Applicata and Springer-Verlag GmbH Germany, part of Springer Nature 2018

\begin{abstract}
We consider the Navier-Stokes equation on the 2D torus, with a stochastic forcing term which is a cylindrical fractional Wiener noise of Hurst parameter $H$. Following Albeverio and Ferrario (Ann Probab 32(2):1632-1649, 2004) and Da Prato and Debussche (J Funct Anal 196(1):180-210, 2002) which dealt with the case $H=\frac{1}{2}$, we prove a local existence and uniqueness result when $\frac{7}{16}<H<\frac{1}{2}$ and a global existence and uniqueness result when $\frac{1}{2}<H<1$.
\end{abstract}

Keywords Stochastic partial differential equation - Navier-Stokes equations · Cylindrical fractional Brownian motion

Mathematics Subject Classification $60 \mathrm{H} 15 \cdot 35 \mathrm{R} 60 \cdot 60 \mathrm{H} 30 \cdot 76 \mathrm{D} 05$

\section{Introduction}

An incompressible fluid flow dynamics is described by the so-called incompressible NavierStokes equations. In this paper, we consider the Navier-Stokes equations on the torus, i.e., we work on the square $\mathbb{T}=[0,2 \pi]^{2}$ with periodic boundary conditions; we add a stochastic forcing term. These are the equations

Benedetta Ferrario is partially supported by INdAM-GNAMPA, by MIUR-Dipartimenti di Eccellenza Program (2018-2022) and by PRIN 2015 "Deterministic and stochastic evolution equations". Christian Olivera is partially supported by FAPESP_Grants 2017/17670-0 and 2015/07278-0.

$凶 \quad$ Benedetta Ferrario

benedetta.ferrario@unipv.it

Christian Olivera

colivera@ime.unicamp.br

1 Dipartimento di Matematica "F. Casorati”, Università di Pavia, Pavia, Italy

2 Departamento de Matemática, Universidade Estadual de Campinas, Campinas, Brazil 


$$
\left\{\begin{array}{l}
\partial_{t} v=v \Delta v-(v \cdot \nabla) v-\nabla p+\partial_{t} W^{H} \\
\operatorname{div} v=0 \\
\left.v\right|_{t=0}=v_{0}
\end{array},\right.
$$

where for $t \in[0, T]$ and $\xi \in \mathbb{T}, v=v(t, \xi)$ is the vector velocity, $p=p(t, \xi)$ the scalar pressure, $v>0$ the viscosity coefficient, and $W^{H}=W^{H}(t, \xi)$ a cylindrical fractional Brownian process.

Stochastic perturbations in the equations of motions are commonly used to model small perturbations (numerical, empirical, and physical uncertainties) or thermodynamic fluctuations present in fluid flows. We refer to the lecture notes by Flandoli [14], the monograph of Kuksin and Shirikyan [17] as well as the references cited therein for a recent overview.

Different noise terms have been considered so far. The contribution of this paper is to study Eq. (3) with a cylindrical fraction Brownian motion $W^{H}$ for $H \neq \frac{1}{2}$. Indeed the case $H=\frac{1}{2}$ has been studied in $[1,3,8,9]$. Let us point out that with a colored (not cylindrical) noise, the analysis of Eq. (1) is easier; some results on a more general bidimensional domain can be found in [12]. Moreover, the bigger is $H$ the more regular is the fractional Brownian motion. Hence it is worth to ask whether the cylindrical fractional Brownian motion with $H<\frac{1}{2}$ can be considered; in addition also the analysis for $H>\frac{1}{2}$ is interesting in order to compare the results for different values of the Hurst parameter. In this paper we shall prove local existence and uniqueness of solutions for $\frac{7}{16}<H<\frac{1}{2}$ and global existence and uniqueness for $\frac{1}{2}<H<1$.

As far as the contents of the paper are concerned, in Sect. 2 we introduce the mathematical setting, in Sect. 3 we analyze the linear Stokes problem, whereas Sect. 4 and Sect. 5 analyze the bilinear term and the Navier-Stokes problem, respectively. In Appendices, we present some proofs.

\section{Mathematical setting}

In this section we introduce the basic tools.

\subsection{The spaces}

For a complex number $b=\Re b+i \Im b$, we denote by $\bar{b}$ the complex conjugate $(\bar{b}=\Re B-i \Im b)$ and by $|b|$ the absolute value $\left(|b|=\sqrt{(\Re b)^{2}+(\Im b)^{2}}\right)$.

We consider subspaces of $\mathbb{Z}^{2}$ :

$$
\begin{aligned}
& \mathbb{Z}_{0}^{2}=\left\{k=\left(k^{(1)}, k^{(2)}\right) \in \mathbb{Z}^{2}: k \neq 0\right\} \\
& \mathbb{Z}_{+}^{2}=\left\{k=\left(k^{(1)}, k^{(2)}\right) \in \mathbb{Z}_{0}^{2}: k^{(1)}>0\right\} \cup\left\{k=\left(k^{(1)}, k^{(2)}\right) \in \mathbb{Z}_{0}^{2}: k^{(1)}=0, k^{(2)}>0\right\}
\end{aligned}
$$

and

$$
\mathbb{Z}_{-}^{2}=\mathbb{Z}_{0}^{2} \backslash \mathbb{Z}_{+}^{2}
$$

When $k=\left(k^{(1)}, k^{(2)}\right) \in \mathbb{Z}^{2}$, we denote by $|k|$ the absolute value $\left(|k|=\sqrt{\left(k^{(1)}\right)^{2}+\left(k^{(2)}\right)^{2}}\right)$.

We consider the separable Hilbert space $\mathcal{H}^{0}$ which is the $L^{2}$-closure of the space of smooth vectors which are periodic, zero mean value, and divergence free. Let $\left\{h_{k}\right\}_{k}$ be the basis for $\mathcal{H}^{0}$, given by $h_{k}(\xi)=\frac{1}{2 \pi} \frac{k^{\perp}}{|k|} e^{i k \cdot \xi}$ for $k \in \mathbb{Z}_{0}^{2}$ and $\xi \in \mathbb{T}$. Notice that, for any $k \in \mathbb{Z}_{0}^{2}$, 
$h_{-k}(\xi)=-\bar{h}_{k}(\xi)$ and $\Delta h_{k}=-|k|^{2} h_{k}$. Therefore,

$$
\mathcal{H}^{0}=\left\{v(\xi)=\sum_{k \in \mathbb{Z}_{0}^{2}} v_{k} h_{k}(\xi): v_{-k}=-\bar{v}_{k} \forall k, \sum_{k \in \mathbb{Z}_{0}^{2}}\left|v_{k}\right|^{2}<\infty\right\}
$$

Notice that the complex coefficients $v_{k}$ must satisfy $v_{-k}=-\bar{v}_{k}$ in order to get a real vector $v$.

More generally, for $r \in \mathbb{R}$ we define

$$
\mathcal{H}^{r}=\left\{v(\xi)=\sum_{k \in \mathbb{Z}_{0}^{2}} v_{k} h_{k}(\xi): v_{-k}=-\bar{v}_{k} \forall k, \sum_{k \in \mathbb{Z}_{0}^{2}}|k|^{2 r}\left|v_{k}\right|^{2}<\infty\right\} .
$$

This is a Hilbert space with scalar product

$$
(u, v)_{\mathcal{H}^{r}}=\sum_{k \in \mathbb{Z}_{0}^{2}}|k|^{2 r} u_{k} \bar{v}_{k} .
$$

Following [4], we define the periodic divergence-free vector Sobolev spaces ( $r \in \mathbb{R}, 1 \leq$ $p \leq \infty)$

$$
\mathcal{H}_{p}^{r}=\left\{v=\sum_{k \in \mathbb{Z}_{0}^{2}} v_{k} h_{k}: \sum_{k \in \mathbb{Z}_{0}^{2}} v_{k}|k|^{r} h_{k} \in\left[L^{p}(\mathbb{T})\right]^{2}\right\}
$$

and the periodic divergence-free vector Besov spaces as real interpolation spaces

$$
\begin{aligned}
\mathcal{B}_{p q}^{r}=\left(\mathcal{H}_{p}^{r_{0}}, \mathcal{H}_{p}^{r_{1}}\right)_{\theta, q}, \quad r & \in \mathbb{R}, 1 \leq p, q \leq \infty \\
r & =(1-\theta) r_{0}+\theta r_{1}, \quad 0<\theta<1
\end{aligned}
$$

In particular, $\mathcal{B}_{22}^{r}=\mathcal{H}_{2}^{r}=\mathcal{H}^{r}$. Moreover, (see [4])

$$
\begin{array}{rlrl}
\|v\|_{\mathcal{B}_{p q_{1}}^{s}} & \leq\|v\|_{\mathcal{B}_{p q_{2}}^{s}} & & \text { for } q_{2} \leq q_{1} \\
\|v\|_{\mathcal{B}_{p q}^{s_{1}}} & \leq\|v\|_{\mathcal{B}_{p q}^{s_{2}}} & & \text { for } s_{1} \leq s_{2} \\
\|v\|_{\mathcal{B}_{p_{1} q}^{s_{1}}} & \leq C\|v\|_{\mathcal{B}_{p_{2} q}^{s_{2}}} & \text { for } s_{1}-\frac{2}{p_{1}}=s_{2}-\frac{2}{p_{2}}
\end{array}
$$

Here $C$ is a generic constant. We make the convention to denote different constants by the same symbol $C$, unless we want to mark them for further reference.

One interesting result in Besov spaces is given by the following estimate of Chemin (see Corollary 1.3.1 in [7]):

$$
\left\|v_{1} v_{2}\right\|_{\mathcal{B}_{p q}^{s}} \leq \frac{C^{s_{1}+s_{2}}}{s_{1}+s_{2}}\left\|v_{1}\right\|_{\mathcal{B}_{p q}^{s_{1}}}\left\|v_{2}\right\|_{\mathcal{B}_{p q}^{s_{2}}}
$$

if

$$
s_{1}+s_{2}>0, \quad s_{1}<\frac{2}{p}, \quad s_{2}<\frac{2}{p}, \quad s=s_{1}+s_{2}-\frac{2}{p}
$$

and $p, q \in[1, \infty]$. 


\subsection{The abstract equation}

Let us consider a unitary viscosity $v=1$ in system (1). Then we write the evolution in abstract form as

$$
\mathrm{d} v(t)=A v(t) \mathrm{d} t-B(v(t), v(t)) \mathrm{d} t+\mathrm{d} w^{H}(t)
$$

with the operators formally defined as $A=\Delta$ and $B(u, v)=P[(u \cdot \nabla) v]$, where $P$ is the projector operator onto the space of divergence-free vector fields. We can represent the stochastic forcing term as

$$
w^{H}(t, \xi)=\sum_{k \in \mathbb{Z}_{0}^{2}} h_{k}(\xi) b_{k}^{H}(t), \quad(t, \xi) \in \mathbb{R} \times \mathbb{T}
$$

where $\left\{b_{k}^{H}\right\}_{k \in \mathbb{Z}_{+}^{2}}$ are a sequence of i.i.d. complex fractional Brownian processes defined on a complete probability space $(\Omega, \mathbb{F}, \mathbb{P})$ with filtration $\left\{\mathbb{F}_{t}\right\}_{\{t \in \mathbb{R}\}}$ and $b_{-k}^{H}=\overline{b_{k}^{H}}$ for all $k \in \mathbb{Z}_{+}^{2}$. We denote by $\mathbb{E}$ the mathematical expectation with respect to $\mathbb{P}$. This means that $b_{k}^{H}(t)=\Re b_{k}^{H}(t)+i \Im b_{k}^{H}(t)$ and $\left\{\Re b_{k}^{H}, \Im b_{k}^{H}(t)\right\}_{k \in \mathbb{Z}_{+}^{2}}$ is a sequence of i.i.d. standard real fractional Brownian processes ( $\mathrm{fBm}$ ) with Hurst parameter $H$. Each element of the sequence is a centered Gaussian process whose covariance is

$$
C(t, s)=\frac{1}{2}\left(|t|^{2 H}+|s|^{2 H}-|t-s|^{2 H}\right)
$$

$w^{H}$ is called an $\mathcal{H}^{0}$-cylindrical fractional Brownian motion, and one can prove that the series (4) converges in any space $U$ with continuous embedding $\mathcal{H}^{0} \subset U$ of Hilbert-Schmidt type.

Now let us define rigorously the operators $A$ and $B$.

The Stokes operator $A$, as a linear operator in $\mathcal{B}_{p q}^{s}$ with domain $\mathcal{B}_{p q}^{s+2}$, generates an analytic semigroup $\left\{e^{t A}\right\}_{t \geq 0}$ in $\mathcal{B}_{p q}^{s}$ and

$$
\left\|e^{t A} v\right\|_{\mathcal{B}_{p q}^{s_{1}}} \leq \frac{C}{t^{\frac{s_{1}-s_{2}}{2}}}\|v\|_{\mathcal{B}_{p q}^{s_{2}}}
$$

for any $t \geq 0, s_{1}>s_{2}$.

As far as the bilinear term $B(u, v)=P[(u \cdot \nabla) v]$ is considered, we recall some basic properties (See [20]). Let $\langle\cdot, \cdot\rangle$ denote the $\mathcal{H}^{-r}-\mathcal{H}^{r}$ duality bracket. One checks by integration by parts that

$$
\left\langle B\left(u_{1}, u_{2}\right), u_{3}\right\rangle=-\left\langle B\left(u_{1}, u_{3}\right), u_{2}\right\rangle
$$

and taking $u_{2}=u_{3}$

$$
\left\langle B\left(u_{1}, u_{2}\right), u_{2}\right\rangle=0 .
$$

These relationships are true with regular entries and then are extended to more general vectors by density.

A basic estimate is (see [15, Lemma 2.2])

$$
\|B(u, v)\|_{\mathcal{H}^{-\delta}} \leq C\|u\|_{\mathcal{H}^{\theta}}\|v\|_{\mathcal{H}^{\rho}}
$$

when

$$
\begin{array}{ll}
0 \leq \delta<2, & \rho>0, \quad \theta>0 \\
\rho+\delta>1, & \theta+\rho+\delta \geq 2
\end{array}
$$


Other estimates have been given before in (2); indeed, by the divergence-free condition, we have $B(u, v)=P[\operatorname{div}(u \otimes v)]$; therefore, $\|B(u, v)\|_{\mathcal{B}_{p q}^{s}} \leq\|u \otimes v\|_{\mathcal{B}_{p q}^{s+1}}$.

Moreover, as done in [3], we can develop the bilinear term in Fourier series. Given $v=$ $\sum_{l \in \mathbb{Z}_{0}^{2}} v_{l} h_{l}$ and $u=\sum_{h \in \mathbb{Z}_{0}^{2}} u_{h} h_{h}$, we have formally

$$
\begin{aligned}
B(u, v) & =i P \sum_{h, l \in \mathbb{Z}_{0}^{2}} u_{h} \frac{h^{\perp} \cdot l}{|h|} \frac{e^{i h \cdot \xi}}{2 \pi} v_{l} \frac{l^{\perp}}{|l|} \frac{e^{i l \cdot \xi}}{2 \pi} \\
& =i P \sum_{k \in \mathbb{Z}_{0}^{2}}\left(\sum_{\substack{h \in \mathbb{Z}_{0}^{2} \\
h \neq k}} \frac{h^{\perp} \cdot k}{2 \pi|h||k-h|} u_{h} v_{k-h}(k-h)^{\perp}\right) \frac{e^{i k \cdot \xi}}{2 \pi} .
\end{aligned}
$$

Using that the projector $P$ acts on the $k$ th component as $P_{k} a=\frac{a \cdot k^{\perp}}{|k|^{2}} k^{\perp}$, we get

$$
B(u, v)=i \sum_{k \in \mathbb{Z}_{0}^{2}}\left(\sum_{\substack{h \in \mathbb{Z}_{0}^{2} \\ h \neq k}} \frac{h^{\perp} \cdot k}{2 \pi|h||k-h|} u_{h} v_{k-h} \frac{(k-h)^{\perp} \cdot k^{\perp}}{|k|}\right) k^{\perp} \frac{e^{i k \cdot \xi}}{2 \pi|k|} .
$$

Summing up, the bilinear term can be written in Fourier series as

$$
B(u, v)=\sum_{k \in \mathbb{Z}_{0}^{2}} B_{k}(u, v) h_{k}
$$

with

$$
\begin{aligned}
B_{k}(u, v) & =i \sum_{\substack{h \in \mathbb{Z}_{0}^{2} \\
h \neq k}} \gamma_{h, k} u_{h} v_{k-h} \\
\gamma_{h, k} & =\frac{1}{2 \pi} \frac{\left(h^{\perp} \cdot k\right)([k-h] \cdot k)}{|h||k-h||k|} .
\end{aligned}
$$

Notice that $\bar{B}_{k}=-B_{-k}$. The convergence of the series (9) will be analyzed in the next section.

Our aim is to study Eq. (3) for $H \neq \frac{1}{2}$. Indeed, the case $H=\frac{1}{2}$ has been studied in $[1,3,8,9]$ : Da Prato and Debussche proved the existence of a strong mild solution for $\mu$-a.e. initial condition (where $\mu$ is the Gibbs measure of the enstrophy, introduced in [2] which is an invariant measure for Eq. (3)), whereas Albeverio and Ferrario proved pathwise uniqueness of these solutions.

We shall prove a local existence and uniqueness result for $\frac{7}{16}<H<\frac{1}{2}$ and a global existence and uniqueness result for $H>\frac{1}{2}$. This latter result improves that of [12]; indeed, the case of cylindrical fBm is included in [12] but only for $H>\frac{3}{4}$. (See Theorem 5.1 and Corollary 4.3 there.) By the way, there are other differences with respect to [12]: in [12] the spatial domain is not the torus but a generic smooth bounded subset $D$ of $\mathbb{R}^{2}$ (and the Dirichlet boundary condition is assumed), and the solution is a process with values in $L^{4}$ in time and space, whereas our solution is more regular since a.a. paths are at least in $C\left([0, T] ; \mathcal{H}^{\frac{1}{2}}\right.$ ) (see next Theorem 2 with $H>\frac{3}{4}$ ) and one knows that $\mathcal{H}^{\frac{1}{2}} \subset L^{4}(D)$. 
In order to analyze Eq. (3), we introduce as in [8] two subproblems: the linear Stokes equation

$$
\mathrm{d} z(t)=A z(t) \mathrm{d} t+\mathrm{d} w^{H}(t)
$$

and the equation for $u=v-z$

$$
\frac{\mathrm{d} u}{\mathrm{~d} t}(t)=A u(t)-B(u(t), u(t))-B(u(t), z(t))-B(z(t), u(t))-B(z(t), z(t))
$$

which is a Navier-Stokes-type equation with random coefficients.

First we deal with the linear problem for $z$, then we define the bilinear term $B(z, z)$ a.s. as in $[3,8]$ and finally face the nonlinear equation for $u$. At the end, we recover the existence result for $v$ from the representation $v=z+u$.

\section{The Stokes equation}

If we neglect the bilinear term in (3), we obtain the linear Stokes equation

$$
\mathrm{d} v(t)=A v(t) \mathrm{d} t+\mathrm{d} w^{H}(t) .
$$

We consider its stationary mild solution; this is the process

$$
z(t)=\int_{-\infty}^{t} e^{(t-s) A} \mathrm{~d} w^{H}(s) .
$$

We can write

$$
\begin{aligned}
z(t)(\xi)= & \sum_{k \in \mathbb{Z}_{0}^{2}} h_{k}(\xi) \int_{-\infty}^{t} e^{-|k|^{2}(t-s)} \mathrm{d} b_{k}^{H}(s) \\
\equiv & 2 \sum_{k \in \mathbb{Z}_{+}^{2}} \frac{k^{\perp}}{2 \pi|k|} \cos (k \cdot \xi) \int_{-\infty}^{t} e^{-|k|^{2}(t-s)} \mathrm{d} \Re b_{k}^{H}(s) \\
& -2 \sum_{k \in \mathbb{Z}_{+}^{2}} \frac{k^{\perp}}{2 \pi|k|} \sin (k \cdot \xi) \int_{-\infty}^{t} e^{-|k|^{2}(t-s)} \mathrm{d} \Im b_{k}^{H}(s) .
\end{aligned}
$$

First, we provide a result for each stochastic convolution integral appearing in the Fourier series representation.

Lemma 1 Let $\lambda>0$ and $b^{H}$ be a real fBm of Hurst parameter $H \in(0,1)$. Then

$$
\int_{-\infty}^{t} e^{-\lambda(t-s)} \mathrm{d} b^{H}(s), \quad t \in \mathbb{R}
$$

is a stationary centered Gaussian process whose variance is

$$
C_{H} \lambda^{-2 H},
$$

where $C_{H}$ is the positive constant given in (14).

Proof Following the proof of Lemma 4.1 in [12], we have that the random variables

$$
\int_{-\infty}^{t} e^{-\lambda(t-s)} \mathrm{d} b^{H}(s)
$$


and

$$
\int_{0}^{+\infty} e^{-\lambda r} \mathrm{~d} b^{H}(r)
$$

have the same law. Moreover, by self-similarity of the $\mathrm{fBm}$, the latter random variable has the same law as

$$
\lambda^{-H} \int_{0}^{+\infty} e^{-r} \mathrm{~d} b^{H}(r)
$$

Therefore,

$$
\mathbb{E}\left(\int_{-\infty}^{t} e^{-\lambda(t-s)} \mathrm{d} b^{H}(s)\right)^{2}=\lambda^{-2 H} \mathbb{E}\left(\int_{0}^{+\infty} e^{-r} \mathrm{~d} b^{H}(r)\right)^{2} .
$$

We estimate $\mathbb{E}\left(\int_{0}^{+\infty} e^{-r} \mathrm{~d} b^{H}(r)\right)^{2}$ using the representation

$$
\int_{0}^{+\infty} e^{-r} \mathrm{~d} b^{H}(r)=\int_{0}^{+\infty} e^{-r} b^{H}(r) \mathrm{d} r .
$$

This comes from the formula on a finite time interval

$$
\int_{0}^{T} e^{-r} \mathrm{~d} b^{H}(r)=e^{-T} b^{H}(T)+\int_{0}^{T} e^{-r} b^{H}(r) \mathrm{d} r
$$

and the fact that by the law of iterated logarithm (see [5]), we get

$$
\lim _{T \rightarrow+\infty}\left|e^{-T} b^{H}(T)\right|=0 \quad \mathbb{P} \text {-a.s. }
$$

Hence

$$
\begin{aligned}
\mathbb{E}\left(\int_{0}^{+\infty} e^{-r} \mathrm{~d} b^{H}(r)\right)^{2} & =\mathbb{E}\left(\int_{0}^{+\infty} e^{-r} b^{H}(r) \mathrm{d} r\right)^{2} \\
& =\int_{0}^{+\infty} \int_{0}^{+\infty} e^{-r} e^{-s} \frac{r^{2 H}+s^{2 H}-|r-s|^{2 H}}{2} \mathrm{~d} r \mathrm{~d} s
\end{aligned}
$$

By elementary calculations, one shows that the latter integral is finite. We set

$$
C_{H}=\int_{0}^{+\infty} \int_{0}^{+\infty} e^{-r} e^{-s} \frac{r^{2 H}+s^{2 H}-|r-s|^{2 H}}{2} \mathrm{~d} r \mathrm{~d} s .
$$

Now we come back to the stationary process $z$ given in (12). We have the following result

Proposition 1 For any $r<2\left(H-\frac{1}{2}\right)$, we have

$$
z \in C\left(\mathbb{R} ; \mathcal{H}^{r}\right) \quad \mathbb{P} \text {-a.s. }
$$

Proof First we show that for any fixed time, the random variable $z(t) \in \mathcal{H}^{r}, \mathbb{P}$-a.s. Indeed, using (13) and the previous lemma, we have

$$
\begin{aligned}
\mathbb{E}\left[\|z(t)\|_{\mathcal{H}^{r}}^{2}\right] & =\sum_{k \in \mathbb{Z}_{0}^{2}}|k|^{2 r}\left|\int_{-\infty}^{t} e^{-|k|^{2}(t-s)} \mathrm{d} b_{k}^{H}(s)\right|^{2} \\
& =\sum_{k \in \mathbb{Z}_{0}^{2}}|k|^{2 r} 2 \frac{C_{H}}{|k|^{4 H}}
\end{aligned}
$$


The latter series is convergent for $4 H-2 r>2$, i.e., $r<2\left(H-\frac{1}{2}\right)$.

It follows that for any finite $m \geq 1$, we have $z \in L_{\text {loc }}^{m}\left(\mathbb{R} ; \mathcal{H}^{r}\right)$, $\mathbb{P}$-a.s. Indeed, $z(t)$ is a Gaussian random variable; so all the moments are finite, i.e., for any $m \geq 2$, there exists a finite constant $e_{m}$ such that

$$
\mathbb{E}\left[\|z(t)\|_{\mathcal{H}^{r}}^{m}\right]=e_{m}
$$

for any $t$. Moreover, the process $z$ is a stationary process, and by interchanging the integrals, for any $T_{1}<T_{2}$, we get

$$
\mathbb{E}\left[\int_{T_{0}}^{T_{1}}\|z(t)\|_{\mathcal{H}^{r}}^{m} \mathrm{~d} t\right]=\int_{T_{0}}^{T_{1}} \mathbb{E}\left[\|z(t)\|_{\mathcal{H}^{r}}^{m}\right] \mathrm{d} t=e_{m}\left(T_{1}-T_{0}\right)<\infty
$$

Since the expectation is finite, then $\int_{T_{0}}^{T_{1}}\|z(t)\|_{\mathcal{H}^{r}}^{m} \mathrm{~d} t<\infty, \mathbb{P}$-a.s.

The continuity in time of the trajectories has been proved in [11] when $H>\frac{1}{2}$ and in [19] when $H<\frac{1}{2}$.

Remark 1 We see that when $H \leq \frac{1}{2}$, the process $z$ at any fixed time takes values in a distributional space. This is the source of the difficulty in our problem.

Remark 2 From the proof of Proposition 1, we obtain that the process $z$ is a stationary process, and for any time $t$, the law of $z(t)$ is the centered Gaussian measure $\mu^{H} \sim \mathcal{N}\left(0, C_{H}(-A)^{-2 H}\right)$. More precisely, we assign the measure $\mu^{H}$ on the sequences $\left\{\left(\Re v_{k}, \Im v_{k}\right)\right\}_{k \in \mathbb{Z}_{+}^{2}}$ as

$$
\mu^{H}=\otimes_{k \in \mathbb{Z}_{+}^{2}} \mu_{k}^{H}
$$

with

$$
\mathrm{d} \mu_{k}^{H}(x, y)=\frac{|k|^{4 H}}{2 \pi C_{H}} e^{-\frac{|k|^{4 H}}{2 C_{H}}\left(x^{2}+y^{2}\right)} \mathrm{d} x \mathrm{~d} y .
$$

When we identify the space $\mathcal{H}^{r}$ with that of the sequences $\left\{\left(\Re v_{k}, \Im v_{k}\right)\right\}_{k \in \mathbb{Z}_{+}^{2}}$ such that $\sum_{k \in \mathbb{Z}_{+}^{2}}|k|^{2 r}\left[\left(\Re v_{k}\right)^{2}+\left(\Im v_{k}\right)^{2}\right]<\infty$, we get $\mu^{H}\left(\mathcal{H}^{r}\right)=1$ for any $r<2\left(H-\frac{1}{2}\right)$ and $\mu^{H}\left(\mathcal{H}^{r}\right)=0$ for any $r \geq 2\left(H-\frac{1}{2}\right)$ (See [16]). Similarly, $\mu^{H}\left(\mathcal{B}_{p q}^{r}\right)=1$ for any $r<$ $2\left(H-\frac{1}{2}\right)$ and $\mu^{H}\left(\mathcal{B}_{p q}^{r}\right)=0$ for any $r \geq 2\left(H-\frac{1}{2}\right)$.

We finish this section with a result on the deterministic Stokes equation, that will be used in the sequel. Given the deterministic linear problem

$$
\left\{\begin{array}{l}
\frac{\mathrm{d} x}{\mathrm{~d} t}(t)=A x(t)+f(t), \quad t \in(0, T] \\
x(0)=x_{0}
\end{array}\right.
$$

we represent its mild solution as

$$
x(t)=e^{t A} x_{0}+\int_{0}^{t} e^{(t-s) A} f(s) \mathrm{d} s
$$

and we have (see [6, Proposition 4.1], based on [10])

Proposition 2 Let $1<p, q, r<\infty$ and $s \in \mathbb{R}$.

For any $f \in L^{r}\left(0, T ; \mathcal{B}_{p q}^{s}\right)$ and $x_{0} \in \mathcal{B}_{p r}^{s+2-\frac{2}{r}}$, there exists a unique solution $x \in$ $W^{1, r}(0, T) \equiv\left\{x \in L^{r}\left(0, T ; \mathcal{B}_{p q}^{s+2}\right): \frac{\mathrm{d} x}{\mathrm{~d} t} \in L^{r}\left(0, T ; \mathcal{B}_{p q}^{s}\right)\right\}$. 
Moreover, the functions $x, \frac{\mathrm{d} x}{\mathrm{~d} t}$ depend continuously on the data $f$ and $x_{0}$, that is there exists a positive constant $C$ such that

$$
\left(\int_{0}^{T}\left(\|x(t)\|_{\mathcal{B}_{p q}^{s+2}}^{r}+\left\|\frac{\mathrm{d} x}{\mathrm{~d} t}(t)\right\|_{\mathcal{B}_{p q}^{s}}^{r}\right) \mathrm{d} t\right)^{1 / r} \leq\left(C \int_{0}^{T}\|f(t)\|_{\mathcal{B}_{p q}^{s}}^{r} \mathrm{~d} t\right)^{1 / r}+\left\|x_{0}\right\|_{\mathcal{B}_{p r}^{s+2-\frac{2}{r}}}
$$

Finally, the space $W^{1, r}(0, T)$ is continuously embedded into the space $C\left([0, T] ; \mathcal{B}_{p r}^{s+2-\frac{2}{r}}\right)$, that is there exists a positive constant $C$ such that

$$
\|x\|_{C\left([0, T] ; \mathcal{B}_{p r}^{s+2-\frac{2}{r}}\right)} \leq C\|x\|_{W^{1, r}(0, T)}
$$

and therefore the initial condition makes sense.

All the constants depend only on $p, q, r, s$.

\section{The bilinear term}

When we study equation for the auxiliary process $u=v-z$, there appears $B(z, z)$. We analyze the space regularity of this term. Following [1-3,8], we estimate it with respect to the Gaussian measure $\mu^{H}$.

Proposition 3 Let $\frac{1}{4}<H<1$ and

$$
\begin{aligned}
& \rho<4 H-3 \text { if } \frac{1}{4}<H<\frac{1}{2} \\
& \rho<2(H-1) \quad \text { if } \frac{1}{2} \leq H<1
\end{aligned}
$$

Then, for any $m \in \mathbb{N}$

$$
\int\|B(z, z)\|_{\mathcal{H}^{\rho}}^{2 m} \mu^{H}(\mathrm{~d} z)<\infty .
$$

Proof Let us begin to perform computations for $m=1$.

First, we explain why we need the lower bound $H>\frac{1}{4}$. By (10) we have

$$
\begin{aligned}
\int\|B(z, z)\|_{\mathcal{H}^{\rho}}^{2} \mu^{H}(\mathrm{~d} z)=\sum_{k \in \mathbb{Z}_{0}^{2}}|k|^{2 \rho} \int\left|B_{k}(z, z)\right|^{2} \mu^{H}(\mathrm{~d} z) \\
=\sum_{k \in \mathbb{Z}_{0}^{2}}|k|^{2 \rho} \sum_{h, h^{\prime} \in \mathbb{Z}_{0}^{2} ; h, h^{\prime} \neq k} \int \gamma_{h, k} z_{h} z_{k-h} \gamma_{h^{\prime}, k} \overline{z_{h^{\prime}} z_{k-h^{\prime}}} \mu^{H}(\mathrm{~d} z) \\
=\sum_{k \in \mathbb{Z}_{0}^{2}}|k|^{2 \rho} \sum_{h \in \mathbb{Z}_{0}^{2}, h \neq k}\left(\gamma_{h, k}^{2}+\gamma_{h, k} \gamma_{k-h, k}\right) \int\left|z_{h}\right|^{2}\left|z_{k-h}\right|^{2} \mu^{H}(\mathrm{~d} z) \\
=2 \sum_{k \in \mathbb{Z}_{0}^{2}}|k|^{2 \rho} \sum_{h} \gamma_{h, k}^{2} \frac{C_{H}^{2}}{|h|^{4 H}|k-h|^{4 H}}
\end{aligned}
$$

From (10) we have that $\gamma_{h, k}=\gamma_{k-h, k}$ and $\gamma_{h, k}^{2} \leq|k|^{2}$; then we can bound $\int\|B(z, z)\|_{\mathcal{H}^{\rho}}^{2} \mathrm{~d} \mu^{H}(z)$ by

$$
\sum_{k \in \mathbb{Z}_{0}^{2}}|k|^{2 \rho+2} \sum_{h \in \mathbb{Z}_{0}^{2}, h \neq k} \frac{1}{|h|^{4 H}|k-h|^{4 H}}
$$


For any fixed $k$, the latter series (over $h$ ) is convergent if and only if $8 H>2$. Therefore we require

$$
H>\frac{1}{4}
$$

The inner series depends on $k$ as proved in Lemma 5 in Appendix A. Therefore the double series (21) is estimated by

$$
\begin{cases}\sum_{k \in \mathbb{Z}_{0}^{2}}|k|^{2 \rho+2} \frac{1}{|k|^{8 H-2}} & \text { if } \frac{1}{4}<H<\frac{1}{2} \\ \sum_{k \in \mathbb{Z}_{0}^{2}}|k|^{2 \rho+2} \frac{\ln |k|}{|k|^{2}} & \text { if } H=\frac{1}{2} \\ \sum_{k \in \mathbb{Z}_{0}^{2}}|k|^{2 \rho+2} \frac{1}{|k|^{4 H}} & \text { if } H>\frac{1}{2}\end{cases}
$$

The first series converges when $\rho<4 H-3$, the second one when $\rho<-1$ and the third one when $\rho<2 H-2$. This provides the summability (19) under conditions (17), (18).

Now, let us consider higher powers $m>1$. We have that (19) holds also for the other powers, since $\mu^{H}$ is Gaussian and therefore the higher moments are expressed by means of the second moments. For completeness we provide computations for $m=2$ in Appendix B.

Using the stationarity we can write (19) also as

$$
\mathbb{E}\left[\|B(z(t), z(t))\|_{\mathcal{H}^{\rho}}^{2 m}\right]=: \tilde{e}_{m}<\infty
$$

for an $t \in \mathbb{R}$. As an easy consequence, we obtain

$$
\mathbb{E}\left[\int_{t_{0}}^{t_{1}}\|B(z(t), z(t))\|_{\mathcal{H}^{\rho}}^{2 m} \mathrm{~d} t\right]=\left(t_{1}-t_{0}\right) \int\|B(z, z)\|_{\mathcal{H}^{\rho}}^{2 m} \mu^{H}(\mathrm{~d} z)<\infty
$$

for any $\infty<t_{0}<t_{1}<\infty$. Hence

Corollary 1 Let $m \geq 1$ and $T>0$. Choosing $\rho$ as in (17), (18) we get

$$
B(z, z) \in L^{m}\left(0, T ; \mathcal{H}^{\rho}\right)
$$

$\mathbb{P}$-a.s.

Remark 3 Notice that for $\frac{1}{2}<H<1$ the quadratic term $B(z, z)$ is in $L^{2}\left(0, T ; \mathcal{H}^{-1}\right), \mathbb{P}$-a.s.

\section{The nonlinear auxiliary equation}

Let $v$ be the unknown for our equation (3) and let $z$ be the stationary Stokes process given by (12). The process $u=v-z$ solves the equation

$$
\frac{\mathrm{d} u}{\mathrm{~d} t}=A u-B(u, u)-B(u, z)-B(z, u)-B(z, z) .
$$

For $r<2\left(H-\frac{1}{2}\right)$ we have $z(0) \in \mathcal{B}_{p q}^{r}, \mathbb{P}$-a.s. and we take $u(0)=v(0)-z(0)$.

We shall prove that Eq. (22) has a local solution when $\frac{7}{16}<H<\frac{1}{2}$ whereas we have a global result when $\frac{1}{2}<H<1$. This implies results for the unknown $v=z+u$. 


\section{$5.1 \frac{1}{4}<H<\frac{1}{2}$}

We consider a mild solution $u$ to Eq. (22). We want to show local existence (and uniqueness) by means of a fixed point argument. Thus we define the mapping $\mathcal{I}$

$$
\begin{aligned}
{[\mathcal{I}(u)](t)=} & e^{t A} u(0)-\int_{0}^{t} e^{(t-s) A} B(u(s), u(s)) \mathrm{d} s-\int_{0}^{t} e^{(t-s) A} B(z(s), u(s)) \mathrm{d} s \\
& -\int_{0}^{t} e^{(t-s) A} B(u(s), z(s)) \mathrm{d} s-\int_{0}^{t} e^{(t-s) A} B(z(s), z(s)) \mathrm{d} s
\end{aligned}
$$

A fixed point of $\mathcal{I}$ is a mild solution of Eq. (22).

Given $T>0$, let

$$
\mathcal{E}_{T}=L^{\beta}\left(0, T ; \mathcal{B}_{p q}^{\alpha}\right) \cap C\left([0, T] ; \mathcal{B}_{p q}^{\sigma}\right) .
$$

First, we want to show that $\mathcal{I}: \mathcal{E}_{T} \rightarrow \mathcal{E}_{T}$ for suitable values of the parameters $\alpha, \beta, \sigma, p, q, H$.

Define

$$
I_{0}(t)=e^{t A} u_{0} .
$$

Given $u_{0} \in \mathcal{B}_{p q}^{\sigma}$, it is an easy result that $I_{0} \in \mathcal{E}_{T}$ when

$$
\alpha<\sigma+\frac{2}{\beta} \text {. }
$$

Indeed, $\left\|e^{t A} u_{0}\right\|_{\mathcal{B}_{p q}^{\sigma}} \leq\left\|u_{0}\right\|_{\mathcal{B}_{p q}^{\sigma}}$; by (5) we have

$$
\int_{0}^{T}\left\|e^{t A} u_{0}\right\|_{\mathcal{B}_{p q}^{\alpha}}^{\beta} \mathrm{d} t \leq C\left\|u_{0}\right\|_{\mathcal{B}_{p q}^{\sigma}}^{\beta} \int_{0}^{T} \frac{\mathrm{d} t}{t^{\frac{\alpha-\sigma}{2} \beta}}
$$

and the latter integral is finite when $\alpha<\sigma+\frac{2}{\beta}$.

To study the integrals involving $B(u, u), B(z, u)$ and $B(u, z)$ we define

$$
I_{1}(u, \tilde{u})(t)=\int_{0}^{t} e^{(t-s) A} B(u(s), \tilde{u}(s)) \mathrm{d} s .
$$

Lemma 2 Let $\alpha, \sigma \in \mathbb{R}$ and $\beta, p, q \geq 1$ be such that

$$
\left\{\begin{array}{l}
\frac{2}{p}+\frac{2}{\beta}<\sigma+1 \\
\alpha<\frac{2}{p}, \quad \sigma<\frac{2}{p} \\
\alpha+\sigma>0
\end{array}\right.
$$

If $u \in L^{\beta}\left(0, T ; \mathcal{B}_{p q}^{\alpha}\right)$ and $\tilde{u} \in C\left([0, T] ; \mathcal{B}_{p q}^{\sigma}\right)$, then

$$
\left\|I_{1}(u, \tilde{u})\right\|_{L^{\beta}\left(0, T ; \mathcal{B}_{p q}^{\alpha}\right)} \leq C T^{\frac{1}{2}-\frac{1}{p}+\frac{\sigma}{2}}\|u\|_{L^{\beta}\left(0, T ; \mathcal{B}_{p q}^{\alpha}\right)}\|\tilde{u}\|_{C\left([0, T] ; \mathcal{B}_{p q}^{\sigma}\right)}
$$

and

$$
\left\|I_{1}(\tilde{u}, u)\right\|_{L^{\beta}\left(0, T ; \mathcal{B}_{p q}^{\alpha}\right)} \leq C T^{\frac{1}{2}-\frac{1}{p}+\frac{\sigma}{2}}\|u\|_{L^{\beta}\left(0, T ; \mathcal{B}_{p q}^{\alpha}\right)}\|\tilde{u}\|_{C\left([0, T] ; \mathcal{B}_{p q}^{\sigma}\right)}
$$

where the constant $C$ is independent of the time $T$. 
Proof We consider the first estimate, since the second one is obtained in the same way interchanging $u$ and $\tilde{u}$.

We use (2) with $s_{1}=\alpha$ and $s_{2}=\sigma$ :

$$
\|B(u, \tilde{u})\|_{\mathcal{B}_{p q}^{\alpha+\sigma-\frac{2}{p}-1}} \leq C\|u\|_{\mathcal{B}_{p q}^{\alpha}}\|\tilde{u}\|_{\mathcal{B}_{p q}^{\sigma}}
$$

where $\alpha<\frac{2}{p}, \sigma<\frac{2}{p}$ and $\alpha+\sigma>0$. Then we get that $B(u, \tilde{u}) \in L^{\beta}\left(0, T ; \mathcal{B}_{p q}^{\alpha+\sigma-\frac{2}{p}-1}\right)$.

Moreover

$$
\left\|I_{1}(u, \tilde{u})\right\|_{L^{\beta}\left(0, T ; \mathcal{B}_{p q}^{\alpha}\right)}^{\beta} \leq \int_{0}^{T}\left(\int_{0}^{t}\left\|e^{(t-s) A} B(u(s), \tilde{u}(s))\right\|_{\mathcal{B}_{p q}^{\alpha}} \mathrm{d} s\right)^{\beta} \mathrm{d} t .
$$

Now, we perform estimates using (5) and the Hölder inequality:

$$
\begin{aligned}
& \int_{0}^{t}\left\|e^{(t-s) A} B(u(s), \tilde{u}(s))\right\|_{\mathcal{B}_{p q}^{\alpha}} \mathrm{d} s \\
& \quad \leq \int_{0}^{t} \frac{C}{(t-s)^{\frac{1}{2}+\frac{1}{p}-\frac{\sigma}{2}}}\|B(u(s), \tilde{u}(s))\|_{\mathcal{B}_{p q}^{\alpha+\sigma-1-\frac{2}{p}}} \mathrm{~d} s \\
& \quad \leq C \int_{0}^{t} \frac{1}{(t-s)^{\frac{1}{2}+\frac{1}{p}-\frac{\sigma}{2}}}\|u(s)\|_{\mathcal{B}_{p q}^{\alpha}}\|\tilde{u}(s)\|_{\mathcal{B}_{p q}^{\sigma}} \mathrm{d} s \\
& \quad \leq C\|\tilde{u}\|_{C\left([0, T] ; \mathcal{B}_{p q}^{\sigma}\right)}\left(\int_{0}^{t} \frac{\mathrm{d} s}{(t-s)^{\left(\frac{1}{2}+\frac{1}{p}-\frac{\sigma}{2}\right) \frac{\beta}{\beta-1}}}\right)^{1-\frac{1}{\beta}}\left(\int_{0}^{t}\|u(s)\|_{\mathcal{B}_{p q}^{\alpha}}^{\beta} \mathrm{d} s\right)^{\frac{1}{\beta}} \\
& \quad \leq C t^{\frac{1}{2}-\frac{1}{p}+\frac{\sigma}{2}-\frac{1}{\beta}}\|\tilde{u}\|_{C\left([0, T] ; \mathcal{B}_{p q}^{\sigma}\right)}\|u\|_{L^{\beta}\left(0, T ; \mathcal{B}_{p q}^{\alpha}\right)}
\end{aligned}
$$

Integrating in time over the interval $[0, T]$, we conclude the proof.

Now we consider the other norm for $I_{1}$.

Lemma 3 Let $\alpha, \sigma \in \mathbb{R}$ and $\beta, p, q \geq 1$ be such that

$$
\left\{\begin{array}{l}
\frac{2}{p}+\frac{2}{q}<\alpha+1 \\
\beta \geq q \\
\alpha<\frac{2}{p}, \quad \sigma<\frac{2}{p} \\
\alpha+\sigma>0
\end{array}\right.
$$

If $u \in L^{\beta}\left(0, T ; \mathcal{B}_{p q}^{\alpha}\right)$ and $\tilde{u} \in C\left([0, T] ; \mathcal{B}_{p q}^{\sigma}\right)$, then

$$
\left\|I_{1}(u, \tilde{u})\right\|_{C\left([0, T] ; \mathcal{B}_{p q}^{\sigma}\right)} \leq C T^{\frac{\alpha}{2}+\frac{1}{2}-\frac{1}{p}-\frac{1}{\beta}}\|u\|_{L^{\beta}\left(0, T ; \mathcal{B}_{p q}^{\alpha}\right)}\|\tilde{u}\|_{C\left([0, T] ; \mathcal{B}_{p q}^{\sigma}\right)}
$$

and

$$
\left\|I_{1}(\tilde{u}, u)\right\|_{C\left([0, T] ; \mathcal{B}_{p q}^{\sigma}\right)} \leq C T^{\frac{\alpha}{2}+\frac{1}{2}-\frac{1}{p}-\frac{1}{\beta}}\|u\|_{L^{\beta}\left(0, T ; \mathcal{B}_{p q}^{\alpha}\right)}\|\tilde{u}\|_{C\left([0, T] ; \mathcal{B}_{p q}^{\sigma}\right)}
$$

where the constant $C$ is independent of the time $T$.

Proof First, from the previous proof we know that $B(u, \tilde{u}) \in L^{\beta}\left(0, T ; \mathcal{B}_{p q}^{\alpha+\sigma-\frac{2}{p}-1}\right)$; when $\beta \geq q$ we also have $B(u, \tilde{u}) \in L^{q}\left(0, T ; \mathcal{B}_{p q}^{\alpha+\sigma-\frac{2}{p}-1}\right)$ and Proposition 2 provides $I_{1}(u, \tilde{u}) \in$ $C\left([0, T] ; \mathcal{B}_{p q}^{\alpha+\sigma-\frac{2}{p}+1-\frac{2}{q}}\right)$ and finally we use that $\mathcal{B}_{p q}^{\alpha+\sigma-\frac{2}{p}+1-\frac{2}{q}} \subseteq \mathcal{B}_{p q}^{\sigma}$ when $\frac{2}{p}+\frac{2}{q} \leq \alpha+1$. 
Now, we perform estimates using (5) and the Hölder inequality.

$$
\begin{aligned}
\left\|I_{1}(u, \tilde{u})(t)\right\|_{\mathcal{B}_{p q}^{\sigma}} & \leq \int_{0}^{t}\left\|e^{(t-s) A} B(u(s), \tilde{u}(s))\right\|_{\mathcal{B}_{p q}^{\sigma}} \mathrm{d} s \\
& \leq \int_{0}^{t} \frac{C}{(t-s)^{\frac{1}{2}+\frac{1}{p}-\frac{\alpha}{2}}}\|B(u(s), \tilde{u}(s))\|_{\mathcal{B}_{p q}^{\alpha+\sigma-\frac{2}{p}-1}} \mathrm{~d} s \\
& \leq \int_{0}^{t} \frac{C}{(t-s)^{\frac{1}{2}+\frac{1}{p}-\frac{\alpha}{2}}}\|u(s)\|_{\mathcal{B}_{p q}^{\alpha}}\|\tilde{u}(s)\|_{\mathcal{B}_{p q}^{\sigma}} \mathrm{d} s \\
& \leq\|\tilde{u}\|_{C\left([0, T] ; \mathcal{B}_{p q}^{\sigma}\right)} \int_{0}^{t} \frac{C}{(t-s)^{\frac{1}{2}+\frac{1}{p}-\frac{\alpha}{2}}\|u(s)\|_{\mathcal{B}_{p q}^{\alpha}} \mathrm{d} s} \\
& \leq C\|\tilde{u}\|_{C\left([0, T] ; \mathcal{B}_{p q}^{\sigma}\right)}\|u\|_{L^{\beta}\left(0, T ; \mathcal{B}_{p q}^{\alpha}\right)}\left(\int_{0}^{t} \frac{\mathrm{d} s}{(t-s)^{\frac{\beta}{\beta-1}\left(\frac{1}{2}+\frac{1}{p}-\frac{\alpha}{2}\right)}}\right)^{1-\frac{1}{\beta}}
\end{aligned}
$$

The latter internal is finite when $\left(\frac{\beta}{\beta-1}\right)\left(\frac{1}{2}+\frac{1}{p}-\frac{\alpha}{2}\right)<1$, i.e.,

$$
\frac{2}{\beta}+\frac{2}{p}<\alpha+1
$$

This inequality is true when $\beta \geq q$ and $\frac{2}{q}+\frac{2}{p}<\alpha+1$, that is our assumptions imply (24).

Computing the time integral and taking the supremum over $t \in[0, T]$ we get the required estimate.

For the integral involving $B(z, z)$ we define the process

$$
I_{2}(t)=\int_{0}^{t} e^{(t-s) A} B(z(s), z(s)) \mathrm{d} s, \quad t \geq 0
$$

where $z$ is the Stokes process given in (12).

Lemma 4 Let $\frac{1}{4}<H<\frac{1}{2}, \beta, p \geq 1, q \geq 2$ and $\alpha, \sigma \in \mathbb{R}$ be such that

$$
\alpha \leq \sigma+1, \quad \sigma<4 H-2 .
$$

Then $I_{2} \in \mathcal{E}_{T}, \mathbb{P}$-a.s.

Proof We proceed pathwise. First we show that $I_{2} \in L^{\beta}\left(0, T ; \mathcal{B}_{p q}^{\alpha}\right)$. From Corollary 1 we know that the paths of $B(z, z)$ are in $L^{\beta}\left(0, T ; \mathcal{B}_{p q}^{\sigma-1}\right)$ for any $\beta \geq 1$ and for $\sigma<4 H-2$. Therefore, according to Proposition 2 the paths of $I_{2}$ are in $L^{\beta}\left(0, T ; \mathcal{B}_{p q}^{\sigma+1}\right)$. When $\alpha \leq \sigma+1$, the embedding theorem gives $I_{2} \in L^{\beta}\left(0, T ; \mathcal{B}_{p q}^{\alpha}\right)$.

Now, we show that $I_{2} \in C\left([0, T] ; \mathcal{B}_{p q}^{\sigma}\right)$. Again by Corollary 1 , for any $q \geq 1$ and $\sigma<4 H-2$ the paths of $B(z, z)$ are in $L^{q}\left(0, T ; \mathcal{B}_{p q}^{\sigma-1}\right)$. We bear in mind Proposition 2 and we get that $I_{2} \in C\left([0, T] ; \mathcal{B}_{p q}^{\sigma+1-\frac{2}{q}}\right)$. When $q \geq 2$ this finishes the proof.

Summing up, we have proved estimates for all the terms in the r.h.s. of (23). Let us point out that merging these results we have to satisfy two conditions:

$$
\sigma<2\left(H-\frac{1}{2}\right)
$$


which comes from Proposition 1 and provides $z \in C\left([0, T] ; \mathcal{H}^{\sigma}\right)$, $\mathbb{P}$-a.s., so to apply Lemmas 2 and 3 for the integrals involving $B(z, u)$ and $B(u, z)$, and

$$
\sigma<4 H-2
$$

which comes from Lemma 4 to estimate the integral involving $B(z, z)$.

When $H<\frac{1}{2}$, the latter condition is stronger and we will write only this one in the following.

Proposition 4 Let $\frac{1}{4}<H<\frac{1}{2}, \alpha, \sigma \in \mathbb{R}, \beta, p \geq 1$ and $q \geq 2$ be such that

$$
\begin{aligned}
\frac{2}{p}+\frac{2}{\beta} & <\sigma+1 \\
\frac{2}{p}+\frac{2}{q} & <\alpha+1 \\
\beta & \geq q \\
\alpha & <\frac{2}{p} \\
\sigma & <\frac{2}{p} \\
\alpha+\sigma & >0 \\
\alpha & <\sigma+\frac{2}{\beta} \\
\alpha & \leq \sigma+1 \\
\sigma & <4\left(H-\frac{1}{2}\right)
\end{aligned}
$$

Then, for any finite $T$ we have that $\mathcal{I}: \mathcal{E}_{T} \rightarrow \mathcal{E}_{T}, \mathbb{P}$-a.s.

Remark 4 (How to fulfill conditions) Notice that by condition (33) we have $\sigma<0$ when $H<\frac{1}{2}$. Therefore, condition (30) requires $\alpha>0$.

Moreover, conditions (30) and (32) provide

$$
-\sigma<\alpha \leq \sigma+1
$$

thus it is necessary that $\sigma>-\frac{1}{2}$, i.e., $H>\frac{3}{8}$.

Actually we are going to show that $H$ must be bigger than $\frac{3}{8}$ in order to satisfy all the conditions (25)-(33). Indeed, we write a system equivalent to the previous one. Condition (29) is trivially satisfied when $\sigma<0$ and can be neglected. Taking $\beta=q$, condition (26) is weaker than condition (25), that is (25) implies (26). In addition, since condition (25) requires $\frac{2}{\beta}<1$, we have that condition (32) is weaker than condition (31), that is (31) implies (32). Therefore, in the case $\beta=q$ the previous system of conditions is equivalent to

$$
\begin{gathered}
\frac{2}{p}+\frac{2}{\beta}<\sigma+1 \\
\beta=q
\end{gathered}
$$




$$
\begin{gathered}
\alpha<\frac{2}{p} \\
\alpha+\sigma>0 \\
\alpha<\sigma+\frac{2}{\beta} \\
\sigma<4\left(H-\frac{1}{2}\right)
\end{gathered}
$$

which is simpler to analyze. Let us notice that

$$
-3 \sigma \underset{\text { by (30) }}{<} \alpha+\alpha-\sigma \underset{\text { by (28) and (31) }}{<} \frac{2}{p}+\frac{2}{\beta} \underset{\text { by (25) }}{<} \sigma+1
$$

This sequence of inequalities is meaningful only when $-3 \sigma<\sigma+1$, i.e., $\sigma>-\frac{1}{4}$. Taking into account the last condition (33), we see that in order to fulfill all the above conditions it is necessary that $H>\frac{7}{16}$.

Therefore it is possible to fulfill all the conditions when $\frac{7}{16}<H<\frac{1}{2}$. Setting $H=\frac{7}{16}+c$ with $0<c<\frac{1}{16}$, we can choose for instance

$$
\alpha=\frac{1}{4}-2 c, \quad \sigma=-\frac{1}{4}+3 c, \quad \frac{2}{\beta}=\frac{2}{q}=\frac{1}{2}-4 c, \quad \frac{2}{p}=\frac{1}{4}-c
$$

in order to satisfy (25)-(33).

Now we can prove the local existence result for $u$, proving that $\mathcal{I}$ is a contraction for $T$ small enough.

Proposition 5 Let $\frac{7}{16}<H<\frac{1}{2}$ and the parameters fulfill the conditions (25)-(33). Then, given $u_{0} \in \mathcal{B}_{p q}^{\sigma}$ there exist a stopping time $\left.\left.\tau \in\right] 0, T\right]$ and for $\mathbb{P}$-a.e. $\omega$ a unique mild solution $u(\omega, \cdot)$ of Eq. (22) with values in $C\left([0, \tau(\omega)] ; \mathcal{B}_{p q}^{\sigma}\right) \cap L^{\beta}\left(0, \tau(\omega) ; \mathcal{B}_{p q}^{\alpha}\right)$.

Proof Using the bilinearity of the operator $B$, we get

$$
\begin{aligned}
\mathcal{I}\left(u_{1}\right)(t)-\mathcal{I}\left(u_{2}\right)(t)= & -\int_{0}^{t} e^{(t-s) A} B\left(u_{1}(s), u_{1}(s)-u_{2}(s)\right) \mathrm{d} s \\
& -\int_{0}^{t} e^{(t-s) A} B\left(u_{1}(s)-u_{2}(s), u_{2}(s)\right) \mathrm{d} s \\
& -\int_{0}^{t} e^{(t-s) A} B\left(z(s), u_{1}(s)-u_{2}(s)\right) \mathrm{d} s \\
& -\int_{0}^{t} e^{(t-s) A} B\left(u_{1}(s)-u_{2}(s), z(s)\right) \mathrm{d} s
\end{aligned}
$$

Let us work in the subspace of $\mathcal{E}_{T}$ with $\|u\|_{\mathcal{E}_{T}} \leq M$. The initial data $u(0) \in \mathcal{B}_{p q}^{\sigma}$ is fixed. Therefore, according to Lemmas 2 and 3, we have

$$
\begin{aligned}
& \left\|\mathcal{I}\left(u_{1}\right)-\mathcal{I}\left(u_{2}\right)\right\|_{\mathcal{E}_{T}} \\
& \quad \leq \bar{C}\left(T^{\frac{1}{2}-\frac{1}{p}+\frac{\sigma}{2}}+T^{\frac{\alpha}{2}+\frac{1}{2}-\frac{1}{p}-\frac{1}{\beta}}\right)\left(M+\|z\|_{C\left([0, T] ; \mathcal{B}_{p q}^{\sigma}\right)}\right)\left\|u_{1}-u_{2}\right\|_{\mathcal{E}_{T}}
\end{aligned}
$$


for a suitable constant $\bar{C}$ independent of $T$.

When $T$ is such that

$$
\bar{C}\left(T^{\frac{1}{2}-\frac{1}{p}+\frac{\sigma}{2}}+T^{\frac{\alpha}{2}+\frac{1}{2}-\frac{1}{p}-\frac{1}{\beta}}\right)\left(M+\|z\|_{C\left([0, T] ; \mathcal{B}_{p q}^{\sigma}\right)}\right)<1
$$

the mapping $\mathcal{I}$ is a contraction and hence has a unique fixed point, which is the unique solution of Eq. (22).

Notice that $T$ is a random time, since inequality (35) involves the random process $z$. It can be chosen to be a stopping time.

Since $v=z+u$, we also get existence of a local mild solution $v$ to Eq. (3) where the bilinear term $B(v, v)$ has to be understood as the sum of four terms, that is

$$
\begin{aligned}
\mathrm{d} v(t)-A v(t) \mathrm{d} t= & -B(u(t), u(t)) \mathrm{d} t-B(u(t), z(t)) \mathrm{d} t \\
& -B(z(t), u(t)) \mathrm{d} t-B(z(t), z(t)) \mathrm{d} t+\mathrm{d} w^{H}(t)
\end{aligned}
$$

Theorem 1 Let $\frac{7}{16}<H<\frac{1}{2}$ and the parameters fulfill the conditions (25)-(33). Then, given $v_{0} \in \mathcal{B}_{p q}^{\sigma}$ there exist a stopping time $\left.\left.\tau \in\right] 0, T\right]$ and for $\mathbb{P}$-a.e. $\omega$ a unique mild solution $v(\omega, \cdot)$ of Eq. (36) with values in $C\left([0, \tau(\omega)] ; \mathcal{B}_{p q}^{\sigma}\right)$.

Remark 5 We cannot get a global existence result for $v$ as in $[1,8]$; indeed, when $H=\frac{1}{2}$ the Gaussian measure $\mu^{H}$ defined by (15) is invariant for the Navier-Stokes equation (3). This allows to define $B(v, v)$ and to get global existence. However, when $H \neq \frac{1}{2}$ the measure $\mu^{H}$ is invariant for the Stokes equation (11) but not for the Navier-Stokes equation (3); this depends eventually on the fact that for $0<H<1$ the Gaussian measure $\mu^{H}$ is (formally) invariant for the deterministic Euler dynamics

$$
\frac{\mathrm{d} v}{\mathrm{~d} t}=-B(v, v)
$$

only when $H=\frac{1}{2}$ (and in this case $\mu^{\frac{1}{2}}$ is called the enstrophy measure, see [2]).

\section{$5.2 \frac{1}{2}<H<1$}

When $\frac{1}{2}<H<1$ the $\mathrm{fBm} w^{H}$ and the Stokes process $z$ are more regular and we expect more regularity of the processes $u$ and $v$ too. Actually we can obtain an a priori energy estimate; this will lead to global existence. Let us notice that now we deal with solutions which are weak in the sense of PDE's; for instance the solution $u$ of Eq. (22) has paths at least in $L^{\infty}\left(0, T ; \mathcal{H}^{0}\right) \cap L^{2}\left(0, T ; \mathcal{H}^{1}\right)$ and fulfills for any $t>0$ and any $\varphi \in \mathcal{H}^{1}$

$$
\begin{aligned}
& \langle u(t)-u(0), \varphi\rangle-\int_{0}^{t}\langle A u(s), \varphi\rangle \mathrm{d} s-\int_{0}^{t}\langle B(u(s), \varphi), u(s)\rangle \mathrm{d} s \\
& -\int_{0}^{t}\langle B(u(s), \varphi), z(s)\rangle \mathrm{d} s-\int_{0}^{t}\langle B(z(s), \varphi), u(s)\rangle \mathrm{d} s=-\int_{0}^{t}\langle B(z(s), z(s)), \varphi\rangle \mathrm{d} s
\end{aligned}
$$

$\mathbb{P}$-a.s. This is obtained from (22) by using (6).

Since the paths of the process $u$ are in $L^{\infty}\left(0, T ; \mathcal{H}^{0}\right) \cap L^{2}\left(0, T ; \mathcal{H}^{1}\right)$ and those of $z$ are in $C\left([0, T] ; \mathcal{H}^{\sigma}\right)$ for some $\sigma>0$, then all the terms in the latter relationship are well defined. Let us check the trilinear terms, by using Hölder inequality, interpolation inequality and Sobolev embeddings: 


$$
\begin{aligned}
|\langle B(u(s), \varphi), u(s)\rangle| & \leq\|u(s)\|_{L^{4}}^{2}\|\varphi\|_{\mathcal{H}^{1}} \leq C\|u(s)\|_{\mathcal{H}^{0}}\|u(s)\|_{\mathcal{H}^{1}}\|\varphi\|_{\mathcal{H}^{1}} \\
|\langle B(u(s), \varphi), z(s)\rangle| & \leq\|u(s)\|_{L^{\frac{2}{\sigma}}}\|\varphi\|_{\mathcal{H}^{1}}\|z(s)\|_{L^{1-\sigma}} \text { for } 0<\sigma<1 \\
& \leq C\|u(s)\|_{\mathcal{H}^{1}}\|\varphi\|_{\mathcal{H}^{1}}\|z(s)\|_{\mathcal{H}^{\sigma}}
\end{aligned}
$$

The third trilinear term can be dealt with as with the second term. And finally the latter term is well defined as soon as $B(z(s), z(s)) \in L^{1}\left(0, T ; \mathcal{H}^{-1}\right)$. (See Remark 3.)

Now, let $0<\sigma<2\left(H-\frac{1}{2}\right)$ for $\frac{1}{2}<H<1$. Taking the $\mathcal{H}^{0}$-scalar product of Eq. (22) with $u$, we get the usual energy estimate (See [20]). We make use of (7) and (8):

$$
\begin{aligned}
\frac{1}{2} \frac{\mathrm{d}}{\mathrm{d} t} & \|u(t)\|_{\mathcal{H}^{0}}^{2}+\|\nabla u(t)\|_{L^{2}}^{2} \\
& =-\langle B(u(t)+z(t), u(t)), u(t)\rangle-\langle B(u(t), z(t)), u(t)\rangle-\langle B(z(t), z(t)), u(t)\rangle \\
& =-\langle B(u(t), z(t)), u(t)\rangle-\langle B(z(t), z(t)), u(t)\rangle \\
& \leq\|B(u(t), z(t))\|_{\mathcal{H}^{-1}}\|u(t)\|_{\mathcal{H}^{1}}+\|B(z(t), z(t))\|_{\mathcal{H}^{-1}}\|u(t)\|_{\mathcal{H}^{1}} \\
& \leq C\|u(t)\|_{\mathcal{H}^{1-\sigma}}\|z(t)\|_{\mathcal{H}^{\sigma}}\|u(t)\|_{\mathcal{H}^{1}}+\frac{1}{4}\|u(t)\|_{\mathcal{H}^{1}}^{2}+C\|B(z(t), z(t))\|_{\mathcal{H}^{-1}}^{2}
\end{aligned}
$$

Moreover, by interpolation and Young inequality

$$
\begin{aligned}
\|u\|_{\mathcal{H}^{1-\sigma}}\|z\|_{\mathcal{H}^{\sigma}}\|u\|_{\mathcal{H}^{1}} & \leq C\|u\|_{\mathcal{H}^{0}}^{\sigma}\|u\|_{\mathcal{H}^{1}}^{1-\sigma}\|z\|_{\mathcal{H}^{\sigma}}\|u\|_{\mathcal{H}^{1}} \\
& =C\|u\|_{\mathcal{H}^{0}}^{\sigma}\|u\|_{\mathcal{H}^{1}}^{2-\sigma}\|z\|_{\mathcal{H}^{\sigma}} \\
& \leq \frac{1}{4}\|u\|_{\mathcal{H}^{1}}^{2}+C\|u\|_{\mathcal{H}^{0}}^{2}\|z\|_{\mathcal{H}^{\sigma}}^{\frac{2}{\sigma}}
\end{aligned}
$$

Since $\|u\|_{\mathcal{H}^{1}}^{2}=\|u\|_{\mathcal{H}^{0}}^{2}+\|\nabla u\|_{L^{2}}^{2}$, collecting all the estimates we have found

$$
\frac{\mathrm{d}}{\mathrm{d} t}\|u(t)\|_{\mathcal{H}^{0}}^{2}+\|\nabla u(t)\|_{L^{2}}^{2} \leq C\left(1+\|z\|_{\mathcal{H}^{\sigma}}^{\frac{2}{\sigma}}\right)\|u\|_{\mathcal{H}^{0}}^{2}+C\|B(z(t), z(t))\|_{\mathcal{H}^{-1}}^{2}
$$

According to Remark 3, $B(z, z) \in L^{2}\left(0, T ; \mathcal{H}^{-1}\right)$. Moreover $z \in C\left([0, T] ; \mathcal{H}^{\sigma}\right)$ by Proposition 1. This provides as usual by means of Gronwall Lemma that $u \in L^{\infty}\left(0, T ; \mathcal{H}^{0}\right) \cap$ $L^{2}\left(0, T ; \mathcal{H}^{1}\right), \mathbb{P}$-a.s. The reader can see all the details of this standard procedure in [20]. First one has to work on the finite dimensional approximation and then pass to the limit. By interpolation

$$
u \in L^{\frac{2}{\sigma}}\left(0, T ; \mathcal{H}^{\sigma}\right) .
$$

Hence, $v=z+u \in L^{\infty}\left(0, T ; \mathcal{H}^{0}\right) \cap L^{\frac{2}{\sigma}}\left(0, T ; \mathcal{H}^{\sigma}\right)$.

We can improve the estimates, now getting $u \in C\left([0, T] ; \mathcal{H}^{\sigma}\right) \cap L^{2}\left(0, T ; \mathcal{H}^{1+\sigma}\right)$. This gives global existence for the process $v=z+u$ in the space $C\left([0, T] ; \mathcal{H}^{\sigma}\right)$ for $0<\sigma<$ $2\left(H-\frac{1}{2}\right)$. Therefore the term $B(v, v)$ is well defined. Actually the process $v$ is a weak solution (in the sense of PDE's) to Eq. (3), that is it solves for any $t>0$ and any $\varphi \in \mathcal{H}^{2-\sigma}$

$$
\left\langle v(t)-v_{0}, \varphi\right\rangle-\int_{0}^{t}\langle A v(s), \varphi\rangle \mathrm{d} s-\int_{0}^{t}\langle B(v(s), \varphi), v(s)\rangle \mathrm{d} s=\left\langle w^{H}(t), \varphi\right\rangle
$$

$\mathbb{P}$-a.s. We leave to the reader to check that all term are well defined (use that $2-\sigma>1$ ). Similarly, the process $z$ can be considered as a weak solution of the stochastic Stokes equation (11). 
Theorem 2 (Global existence) Let $\frac{1}{2}<H<1$ and

$$
0<\sigma<2\left(H-\frac{1}{2}\right) \text {. }
$$

Given $v_{0} \in \mathcal{H}^{\sigma}$ there exists a $C\left([0, T] ; \mathcal{H}^{\sigma}\right) \cap L^{2}\left(0, T ; \mathcal{H}^{1+\sigma}\right)$-valued process $u$ solving Eq. (22) with $u(0)=v_{0}-z(0)$. Therefore there exists a $C\left([0, T] ; \mathcal{H}^{\sigma}\right)$-valued process $v$ solving Eq. (3) with $v(0)=v_{0}$.

Proof We have to work on

$$
\begin{aligned}
\frac{1}{2} \frac{\mathrm{d}}{\mathrm{d} t}\|u(t)\|_{\mathcal{H}^{\sigma}}^{2}+\|\nabla u(t)\|_{H^{\sigma}}^{2} \\
=-\left\langle B(u(t)+z(t), u(t)),(-A)^{\sigma} u(t)\right\rangle-\left\langle B(u(t), z(t)),(-A)^{\sigma} u(t)\right\rangle \\
\quad-\left\langle B(z(t), z(t)),(-A)^{\sigma} u(t)\right\rangle
\end{aligned}
$$

We performed the estimates on the terms in the r.h.s. Notice that $0<\sigma<1$.

From (8), the interpolation inequality $\|u\|_{\mathcal{H}^{1}} \leq C\|u\|_{\mathcal{H}^{\sigma}}^{\sigma}\|u\|_{\mathcal{H}^{1+\sigma}}^{1-\sigma}$ and Young inequality we get

$$
\begin{aligned}
\left\langle B(u, u),(-A)^{\sigma} u\right\rangle & \leq\|B(u, u)\|_{\mathcal{H}^{\sigma-1}}\|u\|_{\mathcal{H}^{\sigma+1}} \\
& \leq C\|u\|_{\mathcal{H}^{\sigma}}\|u\|_{\mathcal{H}^{1}}\|u\|_{\mathcal{H}^{1+\sigma}} \\
& \leq C\|u\|_{\mathcal{H}^{\sigma}}^{1+\sigma}\|u\|_{\mathcal{H}^{1+\sigma}}^{2-\sigma} \\
& \leq \frac{1}{8}\|u\|_{\mathcal{H}^{1+\sigma}}^{2}+C\|u\|_{\mathcal{H}^{\sigma}}^{\frac{2}{\sigma}}\|u\|_{\mathcal{H}^{\sigma}}^{2} \\
\left\langle B(z, u),(-A)^{\sigma} u\right\rangle & \leq\|B(z, u)\|_{\mathcal{H}^{\sigma-1}}\|u\|_{\mathcal{H}^{\sigma+1}} \\
& \leq C\|z\|_{\mathcal{H}^{\sigma}}\|u\|_{\mathcal{H}^{1}}\|u\|_{\mathcal{H}^{1+\sigma}}^{1} \\
& \leq C\|z\|_{\mathcal{H}^{\sigma}}\|u\|_{\mathcal{H}^{\sigma}}^{\sigma}\|u\|_{\mathcal{H}^{1+\sigma}}^{2-\sigma} \\
\left\langle B(u, z),(-A)^{\sigma} u\right\rangle & \leq\|B(u, z)\|_{\mathcal{H}^{\sigma-1}}\|u\|_{\mathcal{H}^{\sigma+1}} \\
& \leq C\|u\|_{\mathcal{H}^{1}}\|z\|_{\mathcal{H}^{\sigma+\epsilon}}\|u\|_{\mathcal{H}^{1+\sigma}}^{2} \\
& \leq C\|u\|_{\mathcal{H}^{\sigma}}^{\sigma}\|z\|_{\mathcal{H}^{\sigma+\epsilon}}\|u\|_{\mathcal{H}^{1+\sigma}}^{2-\sigma}+C\|z\|_{\mathcal{H}^{\sigma}}^{\frac{2}{\sigma}}\|u\|_{\mathcal{H}^{\sigma}}^{2} \\
& \leq \frac{1}{8}\|u\|_{\mathcal{H}^{1+\sigma}}^{2}+C\|z\|_{\mathcal{H}^{\sigma+\epsilon}}^{\frac{2}{\sigma}}\|u\|_{\mathcal{H}^{\sigma}}^{2}
\end{aligned}
$$

where $0<\epsilon \ll 1$, and

$$
\begin{aligned}
\left\langle B(z, z),(-A)^{\sigma} u\right\rangle & \leq\|B(z, z)\|_{\mathcal{H}^{\sigma-1}}\|u\|_{\mathcal{H}^{\sigma+1}} \\
& \leq \frac{1}{8}\|u\|_{\mathcal{H}^{1+\sigma}}^{2}+C\|B(z, z)\|_{\mathcal{H}^{\sigma-1}}^{2}
\end{aligned}
$$

Now, $\|u\|_{\mathcal{H}^{1+\sigma}}^{2}=\|u\|_{\mathcal{H}^{\sigma}}^{2}+\|\nabla u\|_{H^{\sigma}}^{2}$. Summing up

$$
\frac{\mathrm{d}}{\mathrm{d} t}\|u(t)\|_{\mathcal{H}^{\sigma}}^{2}+\|\nabla u(t)\|_{H^{\sigma}}^{2} \leq C\left(1+\|u\|_{\mathcal{H}^{\sigma}}^{\frac{2}{\sigma}}+\|z\|_{\mathcal{H}^{\sigma+\epsilon}}^{\frac{2}{\sigma}}+\|B(z, z)\|_{\mathcal{H}^{\sigma-1}}^{2}\right)\|u\|_{\mathcal{H}^{\sigma}}^{2} .
$$

Now we bear in mind (37), Proposition 1 with $\epsilon \ll 1$ and Proposition 3 to deal with the sum in the r.h.s. So by means of Gronwall lemma, we conclude that $u \in$ 
$L^{\infty}\left(0, T ; \mathcal{H}^{\sigma}\right) \cap L^{2}\left(0, T ; \mathcal{H}^{\sigma+1}\right)$. In addition, by means of the previous estimates we get

$$
\frac{\mathrm{d} u}{\mathrm{~d} t}=A u-B(u, u)-B(u, z)-B(z, u)-B(z, z) \in L^{2}\left(0, T ; \mathcal{H}^{\sigma-1}\right) .
$$

Since $u \in L^{2}\left(0, T ; \mathcal{H}^{\sigma+1}\right)$, one gets that $u \in C\left([0, T] ; \mathcal{H}^{\sigma}\right)$. (See [20].) Hence $v=u+z \in$ $C\left([0, T] ; \mathcal{H}^{\sigma}\right)$.

The solution obtained is also unique. We have a pathwise uniqueness result.

Theorem 3 (Uniqueness) Let $\frac{1}{2}<H<1$ and $0<\sigma<2\left(H-\frac{1}{2}\right)$. Given $v_{0} \in \mathcal{H}^{\sigma}$ there exists a unique $C\left([0, T] ; \mathcal{H}^{\sigma}\right)$-valued process solving (3).

Proof Let $v_{1}, v_{2} \in C\left([0, T] ; \mathcal{H}^{\sigma}\right)$ be solutions of (3). Then the difference $V=v_{1}-v_{2}$ fulfills

$$
\frac{\mathrm{d} V}{\mathrm{~d} t}+A V=-B\left(v_{1}, v_{1}\right)+B\left(v_{2}, v_{2}\right)
$$

with $V(0)=0$. We are going to prove that $V(t)=0$ for all $t \geq 0$ and this is obtained by means of the a priori estimate of the energy. Actually the paths of $V$ are more regular than those of $v_{1}$ and $v_{2}$, since the noise term has disappeared in (39); this was remarked already in [13]. More precisely, we state that any solution $V$ of (39) with $V(0)=0$ is such that $V \in C\left([0, T] ; \mathcal{H}^{0}\right) \cap L^{2}\left(0, T ; \mathcal{H}^{1}\right)$ and $\frac{\mathrm{d} V}{\mathrm{~d} t} \in L^{2}\left(0, T ; \mathcal{H}^{-1}\right)$; therefore the equality $\frac{\mathrm{d}}{\mathrm{d} t}\|V(t)\|_{\mathcal{H}^{0}}^{2}=2\left\langle\frac{\mathrm{d} V}{\mathrm{~d} t}(t), V(t)\right\rangle$ holds and the energy estimates (coming later) are justified.

Indeed we are given $v_{1}, v_{2} \in C\left([0, T] ; \mathcal{H}^{\sigma}\right)$ with $\sigma \in(0,1)$. The r.h.s. of (39) belongs to $L^{\infty}\left(0, T ; \mathcal{H}^{2 \sigma-2}\right)$, since

$$
\left\|B\left(v_{i}, v_{i}\right)\right\|_{\mathcal{H}^{2 \sigma-2}} \leq C\left\|v_{i}\right\|_{\mathcal{H}^{\sigma}}^{2}
$$

by (8). According to Proposition 2 (used with vanishing initial data and $r=2$ ) we get that any solution $V$ will be in $L^{2}\left(0, T ; \mathcal{H}^{2 \sigma}\right)$.

If $2 \sigma \geq 1$ (i.e., when $\left.\frac{1}{2} \leq \sigma<1\right)$ we have obtained that $V \in L^{2}\left(0, T ; \mathcal{H}^{1}\right)$; moreover, $\frac{\mathrm{d} V}{\mathrm{~d} t}=-A V+B\left(v_{1}, v_{1}\right)-B\left(v_{2}, v_{2}\right)$ and therefore $\frac{\mathrm{d} V}{\mathrm{~d} t} \in L^{2}\left(0, T ; \mathcal{H}^{-1}\right)$.

Otherwise, when $0<\sigma<\frac{1}{2}$ we proceed as follows; by the bilinearity of $B$ we get that (39) can be written as

$$
\frac{\mathrm{d} V}{\mathrm{~d} t}+A V=-B\left(v_{1}, V\right)-B\left(V, v_{2}\right)
$$

Let us look at the regularity of the r.h.s., knowing that $v_{1}, v_{2} \in C\left([0, T] ; \mathcal{H}^{\sigma}\right)$ and $V \in$ $C\left([0, T] ; \mathcal{H}^{\sigma}\right) \cap L^{2}\left(0, T ; \mathcal{H}^{2 \sigma}\right)$.

Thanks to (8) we get, for $0<\sigma<\frac{1}{2}$

$$
\begin{aligned}
\left\|B\left(v_{1}, V\right)\right\|_{\mathcal{H}^{3 \sigma-2}} & \leq C\left\|v_{1}\right\|_{\mathcal{H}^{\sigma}}\|V\|_{\mathcal{H}^{2 \sigma}} \\
\left\|B\left(V, v_{2}\right)\right\|_{\mathcal{H}^{3 \sigma-2}} & \leq C\|V\|_{\mathcal{H}^{2 \sigma}}\left\|v_{2}\right\|_{\mathcal{H}^{\sigma}}
\end{aligned}
$$

Hence the r.h.s. of (40) belongs to $L^{2}\left(0, T ; \mathcal{H}^{3 \sigma-2}\right)$ and therefore thanks to Proposition 2 any solution $V$ belongs to $L^{2}\left(0, T ; \mathcal{H}^{3 \sigma}\right)$.

If $3 \sigma \geq 1$ (i.e., when $\left.\frac{1}{3} \leq \sigma<\frac{1}{2}\right)$ we have obtained that $V \in L^{2}\left(0, T ; \mathcal{H}^{1}\right)$ and moreover the r.h.s. of (40) belongs to $L^{2}\left(0, T ; \mathcal{H}^{3 \sigma-2}\right) \subseteq L^{2}\left(0, T ; \mathcal{H}^{-1}\right)$. Hence we conclude as in the previous case about $\frac{\mathrm{d} V}{\mathrm{~d} t}$.

Otherwise, for smaller values of $\sigma$ we proceed again with the bootstrap argument. We conclude that, given $\sigma \in(0,1)$ and $v_{i} \in C\left([0, T] ; \mathcal{H}^{\sigma}\right)$, any solution $V$ to $(40)$ is in $L^{2}\left(0, T ; \mathcal{H}^{1}\right)$ and $\frac{\mathrm{d} V}{\mathrm{~d} t} \in L^{2}\left(0, T ; \mathcal{H}^{-1}\right)$. 
Now, we look for the a priori energy estimate. Keeping in mind (7), (8) and the interpolation inequality $\|V\|_{\mathcal{H}^{1-\sigma}} \leq C\|V(t)\|_{\mathcal{H}^{0}}^{\sigma}\|V(t)\|_{\mathcal{H}^{1}}^{1-\sigma}$, we get

$$
\begin{aligned}
& \frac{1}{2} \frac{\mathrm{d}}{\mathrm{d} t}\|V(t)\|_{\mathcal{H}^{0}}^{2}+\|\nabla V(t)\|_{L^{2}}^{2}=-\left\langle B\left(v_{1}(t), V(t)\right), V(t)\right\rangle-\left\langle B\left(V(t), v_{2}(t)\right), V(t)\right\rangle \\
& =\left\langle B(V(t), V(t)), v_{2}(t)\right\rangle \\
& \leq\|B(V(t), V(t))\|_{\mathcal{H}^{-\sigma}}\left\|v_{2}(t)\right\|_{\mathcal{H}^{\sigma}} \\
& \leq C\|V(t)\|_{\mathcal{H}^{1-\sigma}}\|V(t)\|_{\mathcal{H}^{1}}\left\|v_{2}(t)\right\|_{\mathcal{H}^{\sigma}} \\
& \leq C\|V(t)\|_{\mathcal{H}^{0}}^{\sigma}\|V(t)\|_{\mathcal{H}^{1}}^{2-\sigma}\left\|v_{2}(t)\right\|_{\mathcal{H}^{\sigma}} \\
& \leq \frac{1}{2}\|V(t)\|_{\mathcal{H}^{1}}^{2}+C\left\|v_{2}(t)\right\|_{\mathcal{H}^{\sigma}}^{\frac{2}{\sigma}}\|V(t)\|_{\mathcal{H}^{0}}^{2} \\
& =\frac{1}{2}\|V(t)\|_{\mathcal{H}^{0}}^{2}+\frac{1}{2}\|\nabla V(t)\|_{L^{2}}^{2}+C\left\|v_{2}(t)\right\|_{\mathcal{H}^{\sigma}}^{\frac{2}{\sigma}}\|V(t)\|_{\mathcal{H}^{0}}^{2}
\end{aligned}
$$

From

$$
\frac{\mathrm{d}}{\mathrm{d} t}\|V(t)\|_{\mathcal{H}^{0}}^{2} \leq C\left(1+\left\|v_{2}(t)\right\|_{\mathcal{H}^{\sigma}}^{\frac{2}{\sigma}}\right)\|V(t)\|_{\mathcal{H}^{0}}^{2}
$$

we conclude by Gronwall Lemma that $\sup _{0 \leq t \leq T}\|V(t)\|_{\mathcal{H}^{0}}=0$. This proves pathwise uniqueness.

\section{A Auxiliary results}

We prove some results about convergence of series.

Lemma 5 For any $k \in \mathbb{Z}_{0}^{2}$, the series

$$
\sum_{h \in \mathbb{Z}_{0}^{2}, h \neq k} \frac{1}{|h|^{4 H}|k-h|^{4 H}}
$$

converges if $H>\frac{1}{4}$ and its sum $S_{1}(k)$ depends on $k$ as follows

$$
S_{1}(k) \leq \begin{cases}M_{H} \frac{1}{|k| 8 H-2} & \text { if } \frac{1}{4}<H<\frac{1}{2} \\ M \frac{\ln |k|}{|k|^{2}} & \text { if } H=\frac{1}{2} \\ M_{H} \frac{1}{|k|^{4 H}} & \text { if } H>\frac{1}{2}\end{cases}
$$

for some positive constants $M$ and $M_{H}$ independent of $k$.

Proof The series can be estimated by the following integral

$$
\iint_{D_{k}} \frac{\mathrm{d} x \mathrm{~d} y}{\left[x^{2}+y^{2}\right]^{2 H}\left[\left(x-k^{(1)}\right)^{2}+\left(y-k^{(2)}\right)^{2}\right]^{2 H}}
$$

over the region $D_{k}$ which is the plane without two unitary balls around the points $k$ and 0 . Therefore it is enough to evaluate

$$
I_{1}(a)=\iint_{\tilde{D}_{a}} \frac{\mathrm{d} x \mathrm{~d} y}{\left[x^{2}+y^{2}\right]^{2 H}\left[(x-a)^{2}+y^{2}\right]^{2 H}}
$$

for $a \geq 2$, where $\tilde{D}_{a}=\mathbb{R}^{2} \backslash\left(B_{1}((0,0)) \cup B_{1}((a, 0))\right)$ and $B_{r}((c, d))$ denotes the ball of radius $r$ with center $(c, d)$. 
Now we make a change of variables: $u=\frac{x}{a}$ and $v=\frac{y}{a}$. The domain $D_{a}$ becomes the domain $R_{a}=\mathbb{R}^{2} \backslash\left(B_{\frac{1}{a}}((0,0)) \cup B_{\frac{1}{a}}((1,0))\right)$. Hence

$$
I_{1}(a)=\frac{1}{a^{8 H}} \iint_{R_{a}} \frac{a^{2}}{\left(u^{2}+v^{2}\right)^{2 H}\left((u-1)^{2}+v^{2}\right)^{2 H}} \mathrm{~d} u \mathrm{~d} v .
$$

We split the integral region into three disjoint regions: $R_{a}^{0}=B_{\frac{1}{2}}((0,0)) \backslash B_{\frac{1}{a}}((0,0)), R_{a}^{1}=$ $B_{\frac{1}{2}}((1,0)) \backslash B_{\frac{1}{a}}((1,0))$ and $R^{\infty}=R_{a} \backslash\left(R_{a}^{0} \cup R_{a}^{1}\right)$.

By symmetry the integral over $R_{a}^{0}$ is the same as that over $R_{a}^{1}$ and we have

$$
\begin{aligned}
\iint_{R_{a}^{0}} \frac{\mathrm{d} u \mathrm{~d} v}{\left(u^{2}+v^{2}\right)^{2 H}\left((u-1)^{2}+v^{2}\right)^{2 H}} & \leq \iint_{R_{a}^{0}} \frac{\mathrm{d} u \mathrm{~d} v}{\left(u^{2}+v^{2}\right)^{2 H}\left(\frac{1}{2}\right)^{4 H}} \\
& =2^{4 H} 2 \pi \int_{1 / a}^{1 / 2} \frac{r \mathrm{~d} r}{r^{4 H}} \\
& \leq \begin{cases}\frac{2^{8 H-3} \pi}{\frac{1}{2}-H} & \text { if } \frac{1}{4}<H<\frac{1}{2} \\
8 \pi \ln a & \text { if } H=\frac{1}{2} \\
\frac{2^{4 H-1} \pi}{H-\frac{1}{2}} a^{4 H-2} & \text { if } H>\frac{1}{2}\end{cases}
\end{aligned}
$$

The integral over $R^{\infty}$ is a constant $\tilde{C}_{H}$ independent of $a$, when $H>\frac{1}{4}$. Summing up, we get that there exist positive constants $M$ and $M_{H}$ such that

$$
I_{1}(a) \leq \begin{cases}M_{H} \frac{1}{a^{8 H-2}} & \text { if } \frac{1}{4}<H<\frac{1}{2} \\ M \frac{\ln a}{a^{2}} & \text { if } H=\frac{1}{2} \\ M_{H} \frac{1}{a^{4 H}} & \text { if } H>\frac{1}{2}\end{cases}
$$

Returning to the notation with $k$ we get our result.

In the next lemma, we consider a restricted range for $H$; the assumption on $\rho$ is a restriction of that in (18).

Lemma 6 We are given $\frac{1}{2}<H<1$. Let us assume $-1<\rho<2(H-1)$.

Then, for any $k \in \mathbb{Z}_{0}^{2}$ the series

$$
\sum_{h \in \mathbb{Z}_{0}^{2}, h \neq k} \frac{|h|^{2 \rho+2}}{|h|^{4 H}|k-h|^{4 H}}
$$

converges and its sum $S_{2}(k)$ is bounded by

$$
C_{H} \frac{1}{|k|^{4 H-2 \rho-2}}
$$

for a suitable constant $C_{H}$.

Proof By assumption we have $4 H+4 H-2 \rho-2>2$ and therefore the series is convergent. To estimate its sum we proceed as in the proof of the previous lemma. First the series can be estimated by an integral and it is enough to evaluate

$$
I_{2}(a)=\iint_{\tilde{D}_{a}} \frac{\mathrm{d} x \mathrm{~d} y}{\left[x^{2}+y^{2}\right]^{2 H-\rho-1}\left[(x-a)^{2}+y^{2}\right]^{2 H}}
$$


for $a \geq 2$. By the change of variables $u=\frac{x}{a}$ and $v=\frac{y}{a}$ we obtain

$$
I_{2}(a)=\frac{1}{a^{2(4 H-\rho-1)}} \iint_{R_{a}} \frac{a^{2}}{\left(u^{2}+v^{2}\right)^{2 H-\rho-1}\left((u-1)^{2}+v^{2}\right)^{2 H}} \mathrm{~d} u \mathrm{~d} v .
$$

We split the integral over $R_{a}$ into three parts by setting $R_{a}=R_{a}^{0} \cup R_{a}^{1} \cup R^{\infty}$ with disjoint unions:

- over $R_{a}^{0}=B_{\frac{1}{2}}((0,0)) \backslash B_{\frac{1}{a}}((0,0))$

$$
\begin{aligned}
\iint_{R_{a}^{0}} \frac{\mathrm{d} u \mathrm{~d} v}{\left(u^{2}+v^{2}\right)^{2 H-\rho-1}\left((u-1)^{2}+v^{2}\right)^{2 H}} & \leq 2^{4 H} \iint_{R_{a}^{0}} \frac{\mathrm{d} u \mathrm{~d} v}{\left(u^{2}+v^{2}\right)^{2 H-\rho-1}} \\
& =2^{4 H} 2 \pi \int_{1 / a}^{1 / 2} \frac{r \mathrm{~d} r}{r^{2(2 H-\rho-1)}}
\end{aligned}
$$

By assumption we get that $2(2 H-\rho-1)-1>1$; therefore the latter integral is bounded by

$$
C_{H} a^{4 H-2 \rho-4}
$$

- over $R_{a}^{1}=B_{\frac{1}{2}}((1,0)) \backslash B_{\frac{1}{a}}((1,0))$ we proceed as in the proof of the previous lemma and get that the integral over $R_{a}^{1}$ is bounded by

$$
C_{H} a^{4 H-2}
$$

- over $R^{\infty}=R_{a} \backslash\left(R_{a}^{0} \cup R_{a}^{1}\right)$ : this integral is bounded, uniformly in $a$.

Now we compare the exponents of $a$; since $\rho>-1$, we have $4 H-2 \rho-4<4 H-2$. Summing the three contributions and noticing that $4 H-2>0$ we conclude that the integral over the region $R_{a}$ is bounded by $C_{H} a^{4 H-2}$ for all $a \geq 2$. Thus

$$
I_{2}(a) \leq C_{H} \frac{1}{a^{4 H-2 \rho-2}} \quad \forall a \geq 2
$$

for a suitable constant $C_{H}$.

Lemma 7 Let $\frac{1}{4}<H<1$ and

$$
\begin{gathered}
\rho<4 H-3 \text { if } \frac{1}{4}<H<\frac{1}{2} \\
\rho<2(H-1) \text { if } \frac{1}{2} \leq H<1
\end{gathered}
$$

Then

$$
\sum_{j \in \mathbb{Z}_{0}^{2}}|j|^{2 \rho+2} \sum_{h \neq j} \sum_{l \neq j} \frac{|h-l|^{2 \rho+2}}{|h|^{4 H}|l|^{4 H}|h-j|^{4 H}|l-j|^{4 H}}<\infty .
$$

Proof First let us prove it when $2 \rho+2>0$; this is possible only when $\frac{1}{2}<H<1$. In this case we have

$$
|h-l| \leq 2|h||l|
$$


which holds for any $h, l \in \mathbb{Z}_{0}^{2}$. It comes from

$$
1+\frac{|l|}{|h|} \leq 1+|l| \leq 2|l| \quad \forall|h|,|l| \geq 1
$$

this is equivalent to

$$
|h|+|l| \leq 2|h||l|
$$

Thus, by triangle inequality we obtain (41).

Hence with a positive power we get

$$
|h-l|^{2 \rho+2} \leq C|h|^{2 \rho+2}\left|l^{2 \rho+2}\right|
$$

This implies that we study the series

$$
\sum_{j \in \mathbb{Z}_{0}^{2}}|j|^{2 \rho+2} \sum_{h \neq j} \sum_{l \neq j} \frac{|h|^{2 \rho+2}|l|^{2 \rho+2}}{|h|^{4 H}|l|^{4 H}|h-j|^{4 H}|l-j|^{4 H}}=\sum_{j \in \mathbb{Z}_{0}^{2}}|j|^{2 \rho+2}\left(\sum_{h \neq j} \frac{|h|^{2 \rho+2}}{|h|^{4 H}|h-j|^{4 H}}\right)^{2} .
$$

The inner series is estimated by Lemma 6; thus

$$
\sum_{j \in \mathbb{Z}_{0}^{2}}|j|^{2 \rho+2}\left(\sum_{h \neq j} \frac{|h|^{2 \rho+2}}{|h|^{4 H}|h-j|^{4 H}}\right)^{2} \leq C_{H} \sum_{j \in \mathbb{Z}_{0}^{2}} \frac{1}{|j|^{2(4 H-3 \rho-3)}}
$$

The assumption (18) implies that $2(4 H-3 \rho-3)>2$ and therefore this latter series is convergent.

Now let us consider the case $2 \rho+2 \leq 0$. We have $|h-l|^{(2 \rho+2)} \leq 1$. Therefore we are left with

$$
\sum_{j \in \mathbb{Z}_{0}^{2}}|j|^{2 \rho+2} \sum_{h \neq j} \sum_{l \neq j} \frac{1}{|h|^{4 H}|l|^{4 H}|h-j|^{4 H}|l-j|^{4 H}}=\sum_{j \in \mathbb{Z}_{0}^{2}}|j|^{2 \rho+2}\left(\sum_{h \neq j} \frac{1}{|h|^{4 H}|h-j|^{4 H}}\right)^{2}
$$

We handle this contribution according to Lemma 5. Indeed it is bounded by

$$
\sum_{j \in \mathbb{Z}_{0}^{2}}|j|^{2 \rho+2} \times \begin{cases}M_{H} \frac{1}{|j|^{2(8 H-2)}} & \text { if } \frac{1}{4}<H<\frac{1}{2} \\ M \frac{\ln |j|}{|j|^{4}} & \text { if } H=\frac{1}{2} \\ M_{H} \frac{1}{|j|^{8 H}} & \text { if } H>\frac{1}{2}\end{cases}
$$

Thus there is convergence if

$$
\begin{cases}16 H-4-2 \rho-2>2 & \text { if } \frac{1}{4}<H<\frac{1}{2} \\ 4-2 \rho-2>2 & \text { if } H=\frac{1}{2} \\ 8 H-2 \rho-2>2 & \text { if } H>\frac{1}{2}\end{cases}
$$

These conditions are fulfilled under assumptions (17) and (18). 


\section{B The case $\boldsymbol{m}=\mathbf{2}$}

We present the proof of Proposition 3 for the case $m=2$.

First we shall use many times that $\gamma_{h, k}=\gamma_{k-h, k}$ and $\gamma_{h, k}^{2} \leq|k|^{2}$ for any $h$. We have

$$
\begin{aligned}
& \int\|B(z, z)\|_{\mathcal{H}^{\rho}}^{4} \mu^{H}(\mathrm{~d} z) \\
& =\int\left(\sum_{k \in \mathbb{Z}_{0}^{2}}|k|^{2 \rho}\left|B_{k}(z, z)\right|^{2}\right)^{2} \mu^{H}(\mathrm{~d} z) \\
& =\sum_{k \in \mathbb{Z}_{0}^{2}} \sum_{j \in \mathbb{Z}_{0}^{2}}|k|^{2 \rho}|j|^{2 \rho} \int\left|B_{k}(z, z)\right|^{2}\left|B_{j}(z, z)\right|^{2} \mu^{H}(\mathrm{~d} z) \\
& =\sum_{k \in \mathbb{Z}_{0}^{2}} \sum_{j \in \mathbb{Z}_{0}^{2}}|k|^{2 \rho}|j|^{2 \rho} \sum_{h, h^{\prime} \in \mathbb{Z}_{0}^{2} ; h, h^{\prime} \neq k} \sum_{l, l^{\prime} \in \mathbb{Z}_{0}^{2} ; l, l^{\prime} \neq j} \\
& \int \gamma_{h, k} z_{h} z_{k-h} \gamma_{h^{\prime}, k} \overline{z_{h^{\prime}} z_{k-h^{\prime}}} \gamma_{l, j} z_{l} z_{j-l} \gamma_{l^{\prime}, j} \overline{z_{l^{\prime}} z_{j-l^{\prime}}} \mu^{H}(\mathrm{~d} z)
\end{aligned}
$$

Now we consider the series. There are indeed 6 sums but we have to consider only the non vanishing integrals: $\mu^{H}$ is the product of centered Gaussian measures and all odd powers give zero contribution in the integral with respect to the measure $\mu^{H}$. In particular we analyze

$$
\int z_{h} z_{k-h} \overline{z_{h^{\prime}}} \overline{z_{k-h^{\prime}}} z_{l} z_{j-l} \overline{z_{l^{\prime}}} \overline{z_{j-l^{\prime}}} \mu^{H}(\mathrm{~d} z)
$$

We get different contributions according to the choice of equal indices. Let us list all these possible contributions; we choose $h$ equal to some subsequent index but the case leading to $z_{h}=\overline{z_{k-h}}$ is not possible since $k \neq 0$. Therefore there are 6 possible cases. In the following we do not specify that the sum involves indices belonging to $\mathbb{Z}_{0}^{2}$ in order to shorten the notation.

1. When $h=h^{\prime}$ :

- for $l=l^{\prime}$ we get

$$
\begin{aligned}
& \sum_{k} \sum_{j}|k|^{2 \rho}|j|^{2 \rho} \sum_{h \neq k} \sum_{l \neq j} \int \gamma_{h, k}^{2}\left|z_{h}\right|^{2}\left|z_{k-h}\right|^{2} \gamma_{l, j}^{2}\left|z_{l}\right|^{2}\left|z_{j-l}\right|^{2} \mu^{H}(\mathrm{~d} z) \\
& \quad=\left(\sum_{k}|k|^{2 \rho} \sum_{h \neq k} \int \gamma_{h, k}^{2}\left|z_{h}\right|^{2}\left|z_{k-h}\right|^{2} \mu^{H}(\mathrm{~d} z)\right)^{2}
\end{aligned}
$$

We know from (20) that the quantity in the parenthesis is finite under assumptions (17) and (18).

- for $l=j-l^{\prime}$ we get as before.

2. When $h=k-h^{\prime}$ :

- for $l=l^{\prime}$ we get as before;

- for $l=j-l^{\prime}$ we get as before. 
3. When $h=-l$ we get

$$
\begin{aligned}
& \sum_{k} \sum_{j}|k|^{2 \rho}|j|^{2 \rho} \sum_{h \neq k} \sum_{h^{\prime} \neq k} \sum_{l^{\prime} \neq j} \gamma_{h, k} \gamma_{h^{\prime}, k} \gamma_{-h, j} \gamma_{l^{\prime}, j} \int\left|z_{h}\right|^{2} z_{k-h} \overline{z_{h^{\prime}}} \overline{z_{k-h^{\prime}}} z_{j} \\
& \quad+h \overline{z_{l^{\prime}}} \overline{z_{j}-l^{\prime}} \mu^{H}(\mathrm{~d} z)
\end{aligned}
$$

This gives

- for $k-h=h^{\prime}$ or $h=h^{\prime}$

$$
\begin{aligned}
\sum_{k} & \sum_{j}|k|^{2 \rho}|j|^{2 \rho} \sum_{h \neq k,-j} \sum_{l^{\prime} \neq j} \int \gamma_{h, k}^{2}\left|z_{h}\right|^{2}\left|z_{k-h}\right|^{2} \overline{z_{h}} \gamma_{-h, j} z_{j+h} \gamma_{l^{\prime}, j} \overline{z_{l^{\prime}}} \overline{z_{j-l^{\prime}}} \mu^{H}(\mathrm{~d} z) \\
= & \sum_{k} \sum_{j}|k|^{2 \rho}|j|^{2 \rho} \sum_{h \neq k,-j} \gamma_{h, k}^{2}\left(\gamma_{-h, j}^{2}+\gamma_{-h, j} \gamma_{h+j, j}\right) \\
& \int\left|z_{h}\right|^{4}\left|z_{k-h}\right|^{2}\left|z_{j+h}\right|^{2} \mu^{H}(\mathrm{~d} z) \\
\leq & C \sum_{k} \sum_{j}|k|^{2 \rho+2}|j|^{2 \rho+2} \sum_{h \neq k,-j} \frac{1}{|h|^{8 H}|k-h|^{4 H}|j+h|^{4 H}} \\
= & C \sum_{h} \frac{1}{|h|^{8 H}}\left(\sum_{k \neq h} \frac{|k|^{2 \rho+2}}{|k-h|^{4 H}}\right)^{2}
\end{aligned}
$$

The inner series converges for $\rho<2(H-1)$ (i.e., $2 \rho+2-4 H<-2$ ); from (17) and (18) we know that this condition is fulfilled. Moreover its sum depends on $h$ in such a way that it vanishes when $|h| \rightarrow \infty$; hence it is bounded. Thus we are left with the convergence of the first series $\sum_{h}|h|^{-8 H}$, which holds for any $H>\frac{1}{4}$. So this contribution is finite.

- for $k=-j$

$$
\begin{aligned}
\sum_{k}|k|^{4 \rho} \sum_{h \neq k} \sum_{h^{\prime} \neq k} \sum_{l^{\prime} \neq-k} \int \gamma_{h, k}\left|z_{h}\right|^{2}\left|z_{k-h}\right|^{2} \gamma_{h^{\prime}, k} \overline{z_{h^{\prime}}} \overline{z_{k-h^{\prime}}} \gamma_{-h,-k} \gamma_{l^{\prime},-k} \overline{z_{l^{\prime}}} \overline{z_{-k-l^{\prime}}} \mu^{H}(\mathrm{~d} z) \\
=\sum_{k}|k|^{4 \rho} \sum_{h \neq k} \sum_{h^{\prime} \neq k} \gamma_{h, k} \gamma_{h^{\prime}, k} \gamma_{-h,-k}\left(\gamma_{-h^{\prime},-k}+\gamma_{h^{\prime}-k,-k}\right) \\
\quad \quad \int\left|z_{h}\right|^{2}\left|z_{k-h}\right|^{2}\left|z_{h^{\prime}}\right|^{2}\left|z_{k-h^{\prime}}\right|^{2} \mu^{H}(\mathrm{~d} z) \\
\leq C \sum_{k}|k|^{4 \rho+4} \sum_{h \neq k} \sum_{h^{\prime} \neq k} \frac{1}{|h|^{4 H}|k-h|^{4 H}\left|h^{\prime}\right|^{4 H}\left|k-h^{\prime}\right|^{4 H}} \\
=C \sum_{k}|k|^{4 \rho+4}\left(\sum_{h \neq k} \frac{1}{|h|^{4 H}|k-h|^{4 H}}\right)^{2}
\end{aligned}
$$

We handle this contribution according to Lemma 5. Indeed it is bounded by the series

$$
\sum_{k}|k|^{4 \rho+4} \times \begin{cases}M_{H} \frac{1}{|k|^{8 H-2}} & \text { if } \frac{1}{4}<H<\frac{1}{2} \\ M \frac{\ln |k|}{|k|^{2}} & \text { if } H=\frac{1}{2} \\ M_{H} \frac{1}{|k|^{4 H}} & \text { if } H>\frac{1}{2}\end{cases}
$$


Thus there is convergence if

$$
\begin{cases}8 H-2-4 \rho-4>2 & \text { if } \frac{1}{4}<H<\frac{1}{2} \\ 2-4 \rho-4>2 & \text { if } H=\frac{1}{2} \\ 4 H-4 \rho-4>2 & \text { if } H>\frac{1}{2}\end{cases}
$$

These conditions are fulfilled under assumptions (17) and (18).

- for $k-h=l^{\prime}$

$$
\begin{aligned}
& \sum_{j}|j|^{2 \rho} \sum_{h \neq-j} \sum_{l^{\prime} \neq-h, j} \sum_{h^{\prime} \neq h+l^{\prime}}\left|h+l^{\prime}\right|^{2 \rho} \gamma_{h, h+l^{\prime}} \gamma_{h^{\prime}, h+l^{\prime}} \gamma_{-h, j} \gamma_{l^{\prime}, j} \\
& \quad \int\left|z_{h}\right|^{2}\left|z_{l^{\prime}}\right|^{2} \overline{z_{h^{\prime}}} \overline{z_{h+l^{\prime}-h^{\prime}}} z_{j}+h \overline{z_{j-l^{\prime}}} \mu^{H}(\mathrm{~d} z) \\
& =\sum_{j}|j|^{2 \rho} \sum_{h \neq-j} \sum_{l^{\prime} \neq-h, j}\left|h+l^{\prime}\right|^{2 \rho} \gamma_{h, h+l^{\prime}}\left(\gamma_{h^{\prime}, h+l^{\prime}}+\gamma_{l^{\prime}-j, h+l^{\prime}}\right) \gamma_{-h, j} \gamma_{l^{\prime}, j} \\
& \quad \int\left|z_{h}\right|^{2}\left|z_{l^{\prime}}\right|^{2}\left|z_{j+h}\right|^{2}\left|z_{l^{\prime}-j}\right|^{2} \mu^{H}(\mathrm{~d} z) \\
& \quad \leq C \sum_{j}|j|^{2 \rho+2} \sum_{h \neq-j} \sum_{l^{\prime} \neq-h, j} \frac{\left|h+l^{\prime}\right|^{2 \rho+2}}{|h|^{4 H}\left|l^{\prime}\right|^{4 H}|j+h|^{4 H}\left|l^{\prime}-j\right|^{4 H}} .
\end{aligned}
$$

The convergence of this sums is done in Lemma 7 in Appendix.

- For $k-h=j-l^{\prime}$, we proceed as in the previous case.

4. When $h=l-j$ we get

$$
\begin{gathered}
\sum_{k} \sum_{j}|k|^{2 \rho}|j|^{2 \rho} \sum_{h \neq k,-j} \sum_{h^{\prime} \neq k} \sum_{l^{\prime} \neq j} \gamma_{h, k} \gamma_{h^{\prime}, k} \gamma_{h+j, j} \gamma_{l^{\prime}, j} \\
\int\left|z_{h}\right|^{2} z_{k-h} \overline{z_{h^{\prime}}} \overline{z_{k-h^{\prime}}} z_{h+j} \overline{z_{l^{\prime}}} \overline{z_{j-l^{\prime}}} \mu^{H}(\mathrm{~d} z)
\end{gathered}
$$

Estimating the $\gamma$ 's (that is $\left|\gamma_{, k}\right| \leq C|k|$ ), we get the same computations as in step 3 .

5. When $h=l^{\prime}$, we get the same computations as in step 3 .

6. When $h=j-l^{\prime}$, we get the same computations as in step 3 .

This concludes the proof of (19) for $m=2$.

\section{References}

1. Albeverio, S., Cruzeiro, A.B.: Global flows with invariant (Gibbs) measures for Euler and Navier-Stokes two-dimensional fluids. Commun. Math. Phys. 129(3), 431-444 (1990)

2. Albeverio, S., Ribeiro de Faria, M., Høegh-Krohn, R.: Stationary measures for the periodic Euler flow in two dimensions. J. Stat. Phys. 20(6), 585-595 (1979)

3. Albeverio, S., Ferrario, B.: Uniqueness of solutions of the stochastic Navier-Stokes equation with invariant measure given by the enstrophy. Ann. Probab. 32(2), 1632-1649 (2004)

4. Bergh, L., Löfström, J.: Interpolation Spaces. An introduction. Springer, Berlin (1976)

5. Biagini, F., Hu, Y., Øksendal, B., Zhang, T.: Stochastic Calculus for Fractional Brownian Motion and Applications, Probability and Its Applications (New York). Springer, London (2008)

6. Brzeźniak, Z.: Stochastic partial differential equations in M-type 2 Banach spaces. Potential Anal. 4(1), 1-45 (1995)

7. Chemin J.-Y.: About Navier-Stokes system. Publication du Laboratoire d'Analyse Numérique R 96023 (1996) 
8. Da Prato, G., Debussche, A.: Two-dimensional Navier-Stokes equations driven by a space-time white noise. J. Funct. Anal. 196(1), 180-210 (2002)

9. Debussche, A.: The 2D-Navier-Stokes Equations Perturbed by a Delta Correlated Noise, Probabilistic Methods in Fluids, 115129. World Scientific, River Edge (2003)

10. Dore, G., Venni, A.: On the closedness of the sum of two closed operators. Math. Z. 196(2), 189-201 (1987)

11. Duncan, T.E., Pasik-Duncan, B., Maslowski, B.: Fractional Brownian motion and stochastic equations in Hilbert spaces. Stoch. Dyn. 2(2), 225-250 (2002)

12. Fang, L., Sundar, P., Viens, F.: Two-dimensional stochastic Navier-Stokes equations with fractional Brownian noise. Random Oper. Stoch. Equ. 21(2), 135-158 (2013)

13. Ferrario, B.: Uniqueness result for the 2D Navier-Stokes equation with additive noise. Stoch. Stoch. Rep. 75(6), 435-442 (2003)

14. Flandoli, F.: An introduction to 3D stochastic fluid dynamics. In: SPDE in Hydrodynamic: Recent Progress and Prospects. Lecture Notes in Mathematics, vol. 1942, pp. 51-150. Springer, Berlin (2008)

15. Giga, Y., Miyakawa, T.: Solutions in $L_{r}$ of the Navier-Stokes initial value problem. Arch. Ration. Mech. Anal. 89(3), 267-281 (1985)

16. Kuo, H.H.: Gaussian measures in Banach spaces. In: Lecture Notes in Mathematics, vol. 463. Springer, Berlin (1975)

17. Kuksin, S., Shirikyan, A.: Mathematics of Two-Dimensional Turbulence, vol. 194 of Cambridge Tracts in Mathematics. Cambridge University Press, Cambridge (2012)

18. Maslowski, B., van Neerven, J.: Equivalence of laws and null controllability for SPDEs driven by a fractional Brownian motion. Nonlinear Differ. Equ. Appl. 20, 1473-1498 (2013)

19. Pasik-Duncan, B., Duncan, T.E., Maslowski, B.: Linear stochastic equations in a Hilbert space with a fractional Brownian motion. In: Yan, H., Yin, G., Zhang, Q. (eds.) Stochastic Processes, Optimization, and Control Theory: Applications in Financial Engineering, Queueing Networks, and Manufacturing Systems, International Series in Operations Research \& Management Science, vol. 94, pp. 201-221. Springer, New York (2006)

20. Temam, R.: Navier-Stokes Equations., Theory and Numerical Analysis, Studies in Mathematics and Its Applications, vol. 2. North-Holland, Amsterdam (1977) 